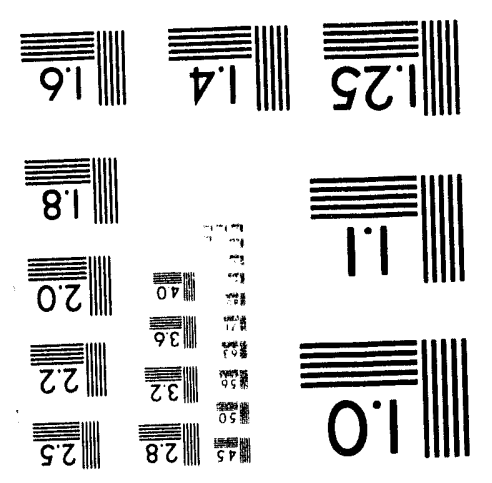



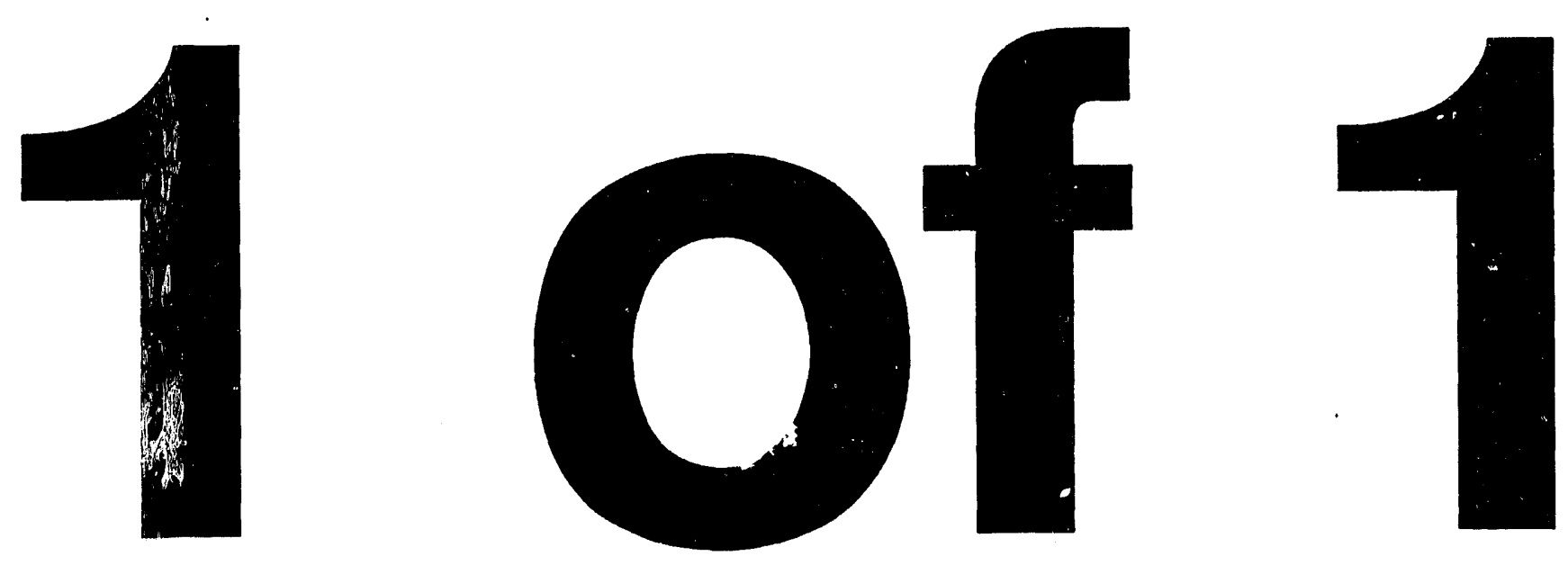


\section{Complex Source Rate Estimation for Atmospheric Transport and Dispersion Models}

\section{Les Edwards}

September 13, 1993

This is an informal report intended primarily for internal or limited external distribution. The opinions and conclusions stated are those of the author and may or may not be those of the Laboratory.

Work performed under the auspices of the U.S. Department of Energy by the Lawrence Livermore National Laboratory under Contract W-7405-Eng-48. 


\section{DISCLAIMER}

This document was prepared as an account of work sponsored by an agency of the United States Government. Neither the United States Government nor the University of California nor any of their employees, makes any warranty, express or implied, or assumes any legal liability or responsibility for the accuracy, completeness, or usefuiness of any information, apparatus, product, or process disclosed, or represents that its use would not infringe privasely owned rights. Reference herein to any specific commercial products, process, or service by trade name, trademark, manufacturer, or otherwise, does not necessarily constitute or imply its endorsement, recommendation, or favoring by the United States Government or the University of Callfornia. The views and opinions of authors expressed herein do not necessarily state or reffect those of the United States Government or the University of California, and shall not be used for advertising or product endorsement purposes.

This report has been reproduced directly from the best available copy.

Available to DOE and DOE contractors from the

Office of Scientific and Technical Information

P.O. Box 62, Oak Ridge, TN 37831

Prices available from (615) 576-8401, FTS 626-8401

Available to the public from the

National Technical Intormation Service

U.S. Department of Commerce

5285 Port Royal Rd.

Springfield, VA 22161 


\title{
COMPLEX SOURCE RATE ESTIMATION FOR ATMOSPHERIC TRANSPORT AND DISPERSION MODELS
}

\author{
Leslie L. Edwards, \\ Lawrence Livermore National Laboratory \\ P.O. Box 808, L-262 \\ Livermore, California, USA 94550
}

\begin{abstract}
The accuracy associated with assessing the environmental consequences of an accidental atmospheric release of radioactivity is highly dependent on our knowledge of the source release rate which is generally poorly known. This paper reports on a technique that integrates the radiological measurements with atmospheric dispersion modeling for more accurate source term estimation. We construct a minimum least squares methodology for solving the inverse problem with no a priori information about the source rate.

The possible ill-posed inverse problem is demonstrated to be non-existent due to what we call "practical regularization" brought about by combinations of the diffusive nature of the atmospheric pollutant and the accuracy of atmospheric measurement instruments. The methodology is tested using simulated data sets which are model generated with random perturbations. We also demonstrate the practical implementation by simulating an emergency response scenario with accidental release of unknown rate.

In general we find that the accuracy with which the algorithms reproduce a known source term is limited only by the amount and accuracy of the measured data. For example, the simulated emergency response scenario has a "double explosion" type of release. Continuous samplers are located at 0.5 and $1.0 \mathrm{~km}$ downwind, and, for 5 minute averaged rates with $30 \%$ random perturbations, if the meteorology is properly guessed, both the reconstructed peak concentrations at 10,20 and $30 \mathrm{~km}$ downwind as well as the estimated total mass released are within $9 \%$ of the actual values. If the meteorology is off by a single Pasquill-Gifford stability category the estimates are still within a factor of 5 . If the diffusion is correct but the wind speed is off by $1 \mathrm{~m} / \mathrm{s}$ the estimates are within $25 \%$. That is, the source term algorithms do a very credible estimation, which, when coupled with the heuristic confidence measures, should prove to be extremely useful for emergency response decision making.
\end{abstract}

\section{MASTER}

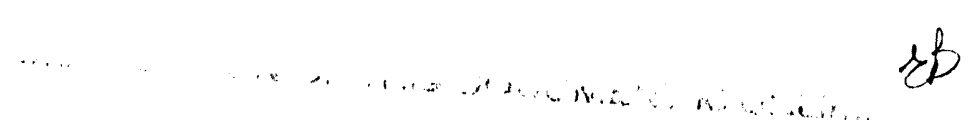




\section{Introduction}

In the event of an accidental atmospheric release of radioactive pollutants, real-time dose assessments are typically derived on the basis of radiological measurements collected throughout the area of interest and/or by atmospheric dispersion modeling. Each of these techniques, taken individually, has limitations for source term as well as individual and collective dose estimation (c.f., $1,2,3,4$ ). The primary limitation associated with the measurements approach is the inability of the local emergency response organization to provide sufficient temporal and spatial coverage in a timely manner for decision making due to limited measurement resources. The uncertainties associated with atmospheric dispersion modeling, on the other hand, largely depend on our ability to simulate the physical processes responsible for pollutant dispersion and the availability of high quality information regarding the release characteristics, the wind, temperature and turbulence structure of the atmosphere, as well as the surface characteristics that affect pollutant behavior. Since our knowledge about these parameters is generally somewhat limited, the model predictions are inherently uncertain especially for situations involving complex source emissions, terrain and meteorology.

These limitations may, to some extent, be overcome by integration of the two techniques to take advantage of their respective strengths. A mathematically sound approach involves optimizing the agreement between the model predicted and the measured concentration distributions by taking advantage of our knowledge of the relative uncertainties associated with the model performance, the various model input parameters and the radiological measurements. This optimization typically involves varying poorly known model input parameters in a selective fashion. The largest uncertainties are generally associated with poorly defined source term characteristics (c.f., $1, \underline{2}, \underline{3}, \underline{5}$ ) such as the location, timing, amount and height of the release; while the temporal and spatial representativeness of the meteorological observations can also be a problem in areas of sparse data coverage. Thus, it becomes a problem of intelligently using all of the complexity of the meteorology and terrain, all of which are assumed known at least to an acceptable degree of uncertainty, the available radiological and meteorological information in a manner consistent with the model's capabilities and limitations to obtain the most accurate representation of the accident scenario and the atmospheric dispersion and deposition processes pertinent to the impact assessment.

In this work we supplement that reported earlier (6) where we demonstrated the estimation of constant (but unknown) release rates. Here we discuss some mathematical aspects of solving the inverse problem including a discussion of "practical regularization" for ill-posed inverse problems.. We improve the algorithms of (으) to demonstrate complex source rate estimation with no a priori information about the source term. The method gives a solution in terms of step functions that approximate the true release rate. We show results of estimations for "simulated" data using a Sequential Gaussian Puff model for atmospheric transport and dispersion.

To demonstrate how the predictor/corrector methodology can be implemented in emergency response situations, we simulate an accidental release where measurements become available in short time increments. We then estimate a complex release rate and predict the expected downwind concentrations. As more measurements become available, the predictions are updated in a real-time manner.

\section{Problem Statement}

Given that an atmospheric release of pollutant has occurred at some given location, $\mathbf{x}_{0}=(\mathrm{x}, \mathrm{y}, \mathrm{z}) \equiv\left(0,0, \mathrm{z}_{0}\right)$, and time, $\mathrm{T}_{0}$, and given a vector, $\mathbf{O}$, of measured concentrations with components 


$$
\begin{aligned}
& \mathrm{o}_{\mathrm{i}}=o\left(\mathrm{x}_{\mathrm{i}}, \mathrm{t}_{\mathrm{i}}\right)=\left\{\text { pollutant concentration, e.g., } / \mathrm{lg} / \mathrm{m}^{3} \text {, taken at location } \mathbf{x}_{\mathrm{i}}\right. \\
& \text { and at time } \left.\mathrm{t}_{\mathrm{i}}>\mathrm{T}_{0} \text { for } \mathrm{i}=1,2, \ldots, \mathrm{I}\right\},
\end{aligned}
$$

the problem is to estimate the pollutant source release rate, $s(\tau)$, say in $\mu g / s$, for $\tau \in\left[t_{0}, T\right] \subseteq\left[T_{0}, T_{1}\right]$, the time of interest, by use of a model of atmospheric transport and dispersion. The choice of this model is made based on the scale of the problem, the complexity of the meteorology and terrain, as well as the nature of the measurement data, all of which are assumed known at least to an acceptable degree of uncertainty.

Any reasonable model of atmospheric transport and dispersion is equivalent to (or, in the case of a numerical model, at least approximately) a continuous and bounded function of time. Thus, we may represent the model computed concentration, in integral form by

$$
c(\mathbf{x}, t)=\int_{T_{0}}^{T_{1}} K(\mathbf{x}, t, \tau) s(\tau) d \tau
$$

where the kernel, $K(\mathbf{x}, t, \tau)$, may be a simple Sequential Gaussian Puff (SGP) model, or a sophisticated numerical model, such as MATHEW/ADPIC (4,2,8,9,10,11).

Our problem may now be stated as: Find $s(\tau)$ or an approximation to $s(\tau)$ such that $\mathrm{c}_{\mathrm{i}}(\mathrm{s}(\tau)) \equiv \mathrm{c}\left(\mathbf{x}_{\mathrm{i}}, \mathrm{t}_{\mathrm{i}}, \mathrm{s}(\tau)\right)$ closely approximates the measurements, $\mathrm{O}_{\mathrm{i}} \equiv \mathrm{o}\left(\mathbf{x}_{\mathrm{i}}, \mathrm{t}_{\mathrm{i}}\right)$ for all $\mathrm{i}$. In the least squares sense, we seek $\hat{s}(\tau)$ such that, if $\Phi$ represents the set of acceptable candidate functions to represent the source term, then

$$
\hat{s}(\tau)=\min _{s(\tau) \in \Phi} \sum_{i=1}^{1}\left[o_{i}-c_{i}(s(\tau))\right]^{2} \equiv \min _{s(\tau) \in \Phi} \sum_{i=1}^{1}\left[R_{i}\right]^{2}
$$

and we would take $\hat{s}(\imath$, as the solution of the inverse problem.

From a practical computational viewpoint, we restrict our set, $\Phi$, of acceptable candidate functions to simple step functions as shown in Fig. 1. We divide the possible release time interval $\left[\mathrm{t}_{0}, \mathrm{~T}\right]$ into $\mathrm{J}$ equal subintervals of length $\Delta \tau \equiv\left(T-\mathrm{t}_{0}\right) / \mathrm{J}$. Let $\tau_{\mathrm{j}}=\mathrm{t}_{0}+\mathrm{j} \Delta \tau$, and, for $\mathrm{j}=1,2, \ldots \mathrm{J}$, define the parameter vector,

$$
\mathbf{P}=\left\{p_{j} \mid p_{j}=\text { approximate rate for } \tau \in\left[\tau_{j-1}, \tau_{j}\right)\right\}
$$

to approximate the actual source rate, $s(\tau)$. We also discretize the transport and dispersion model by computing an IxJ matrix, A, to represent the "unit" response function. That is, the element

$$
\begin{aligned}
a_{i, j} \equiv & \text { model computed concentration at location } x_{i} \text { due to } \\
& \text { a unit release rate during the interval }\left[\tau_{j-1}, \tau_{j}\right)
\end{aligned}
$$


so that, in operator form,

$$
A \mathbf{P} \approx c(x, t, s(\tau))
$$

from which our "least squares inverse" problem is to solve

$$
\mathbf{A P}=\mathbf{O} \text { subject to } \mathbf{P} \geq \mathbf{0} \text {. }
$$

for $\widehat{\mathbf{P}}$, the best step function source rate fit to the data.

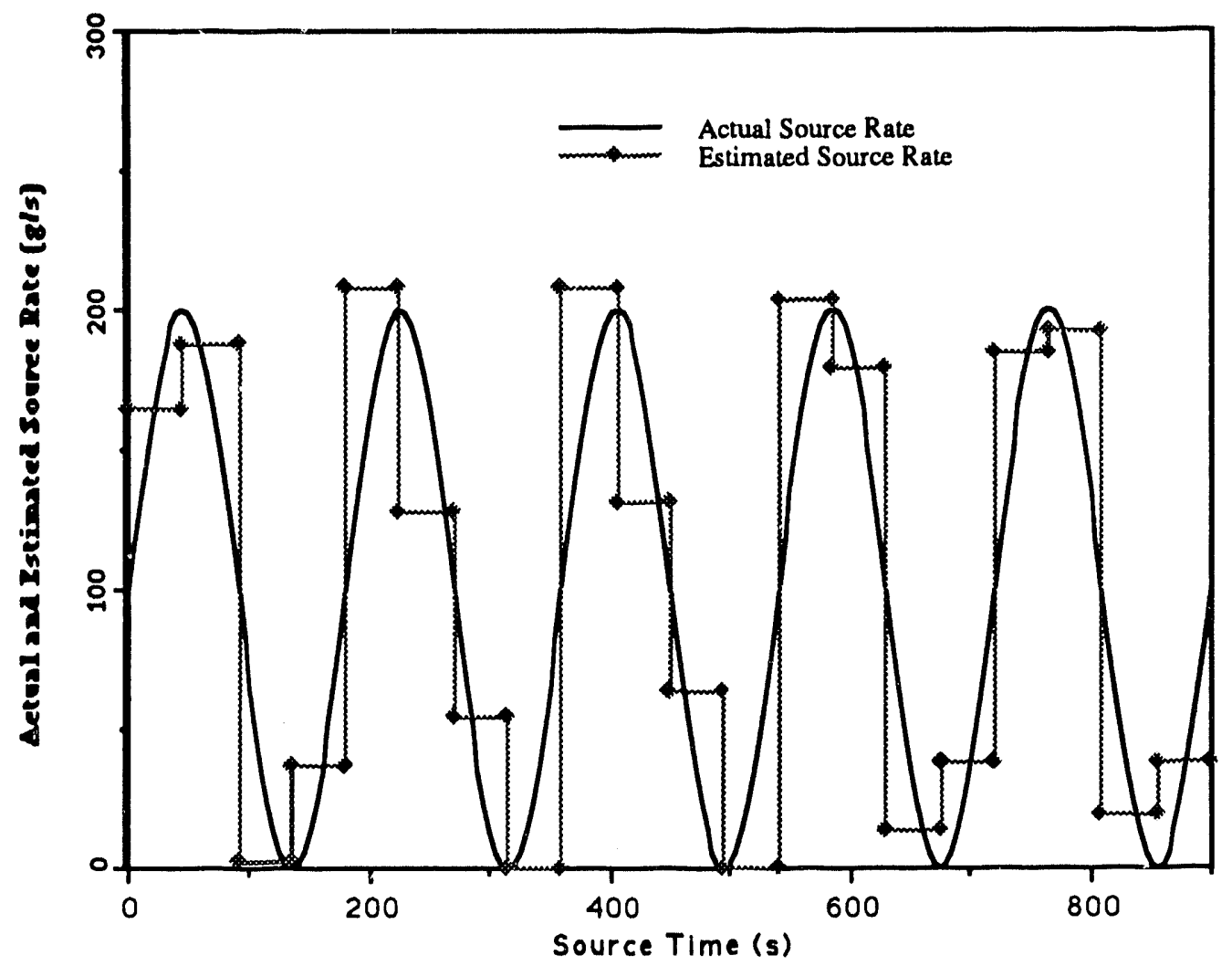

Figure 1: Step-function estimation of source release rate using the subintervals associated with the parameter vector, $\mathbf{P}$.

\section{Mathematical Aspects ${ }^{\mathrm{a}}$ and Practical Regularization}

If all our functions were "mathematically nice" and $\mathbf{A}$ was square (i.e., the number of measurements, $I$, was equal to the number of parameter intervals, $J$ ) and non-singular, then

$$
\hat{\mathbf{P}}=\mathbf{A}^{-1} \mathbf{O}
$$

a The author expresses sincere gratitude to Dr. Sergei E. Pitovranov of the Institute of Systems Studies, Moscow, Russia, for extensive tutorials and discussions concerning the theory of ill-posed problems and the classical methods of regularization. 
approximates the best source term. However, from a mathematical viewpoint, (2) is a Fredholm integral equation of the first kind and it is well known (c.f., 12,13) that such inverse problems may be ill-posed. ${ }^{b}$

The ill-posed nature of an equation has consequences for its numerical treatment even if we view the numerical approximation of a given equation as the solution based on a finite set of perturbed measurements. Straightforward application of numerical methods to ill-posed problems will usually generate numerical nonsense and, often, increasing the degree of discretization on the operator produces approximate solutions to the problem that become less and less reliable $(13, \mathrm{p} 224)$.

Based primarily on the initial work of Tichonov (15), the reduction of an ill-posed problem to a (approximate) well-posed problem is called a regularization of the problem. There exist different approaches for regularization of the problem given by Eq. 2 . Indeed, with the ever increasing computer capability, entire books and conferences are devoted to numerical treatment of integral equations (e.g., 16,17).

In this work we will not be overly concerned with the mathematical aspects of ill-posedness. Rather, we demonstrate that in the "practical application" of source term estimation in the case of atmospheric transport and diffusion, the very nature of diffusion and the precision with which we are able to take measurements of pollutant concentrations provide a practical regularization. We then demonstrate that an acceptable approximate solution to the inverse problems of interest may be obtained.

In terms of "practical" regularization we make the following observations:

1) Given a rapidly time-varying release of pollutant into the atmosphere, then during the transport of the pollutant, atmospheric diffusion will, at some distance downwind, smooth peaks and valleys so that the nature of the original release may be unobservable.

b Definition (J. Hadamard (14)). A mathematical problem is called well-posed if the following three conditions hold: a) A solution of the problem exists; b) the solution is unique; and, c) the solution continuously depends upon input data, i.e., the inverse operator $\mathrm{K}^{-1}$ is continuous on $\mathrm{Y}$. A problem is called ill-posed if any of conditions $\mathrm{a}, \mathrm{b}$, or $\mathrm{c}$ are violated for the specified problem.

For example, the function $s(\tau)=(1+\sin (w \tau)) \in \Phi$, but it does not belong to a set of continuous of the same degree functions when $w \rightarrow \infty$. In fact, the

Theorem (Riemann-Lebesgıe): For an arbitrary integrable function, $f$, defined on [a,b],

$$
\lim _{\omega \rightarrow \infty} \int_{a}^{b} f(t) \cos (w t) d t=\lim _{\omega \rightarrow \infty} \int_{a}^{b} f(t) \sin (w t) d t=0
$$

Application of the theorem to Eq. 2 gives

$$
y(\vec{x}, t)=\int_{0}^{T_{0}} K(\vec{x}, t, \tau)[1+\sin (w \tau)] d \tau=\int_{0}^{T_{0}} K(\vec{x}, t, \tau) d \tau+0=\text { constan } t \text { as } w \rightarrow \infty .
$$

This means that changes in the source term as large as 1 may occur independent of the magnitude of changes in the given function $y$, clearly a violation of Hadamard's third postulate. 
2) If the observed concentrations are either time integrated depositions or time integrated air concentrations, then any perturbations on the source rate that are relatively short in duration relative to the time of sample integration will be lost in the integrated sample;

3) If the observed concentrations are given as a rate (e.g., $\left.\mu \mathrm{g} / \mathrm{m}^{3} / \mathrm{s}\right)$ and these rates are obtained as an average rate over some time period then the same comment applies;

4) Atmospheric pollution measuring devices inherently have some error involved, say 20 to $30 \%$ (c.f., 18) so that it is impossible to distinguish between source rate perturbations and measurement errors when the magnitudes of the perturbations are of the same order as the errors; and

5) For practical implementation, the number of source rate intervals, J, must be reasonably manageable (say 100 or less) in terms of matrix/vector inversions.

Thus, any source rate perturbations that have frequency smaller than $\Delta \tau$ cannot be numerically approximated with much accuracy.

To illustrate the first four points, we employ a Sequential Gaussian Puff (SGP) model (c.f., 4) for which we define the source rate, $s(\tau)$ in $g / s$, by

$$
s(\tau)=N[1+\sin (\pi w \tau / 900)] \text { for } 0 \leq \tau \leq 900 \text { seconds }
$$

where $\mathrm{N}$ is a normalizer so that we release a total of $9 \times 10^{4}$ grams. Figure 2 shows the function and its iniegral for $w=0,1$ and 10.

The pollutant is assumed to be gaseous (i.e., no fall velocity) and released $10 \mathrm{~m}$ above ground level. We then suppose that we have 60 ground level sampling instruments that can be turned on at $20 \mathrm{~s}$ intervals to exactly measure deposition rates in $\mu \mathrm{g} / \mathrm{m}^{2} / \mathrm{s}$. For our purposes in this section we will place all 60 instruments at the same location downwind on the centerline of the deposition pattern. We consider two cases of atmospheric transport and diffusion. The first case has a moderately unstable atmosphere (Pasquill-Gifford category B) with a constant wind speed of $4 \mathrm{~m} / \mathrm{s}$. The second case has slightly stable conditions (category $\mathrm{E}$ ) with wind speed of $10 \mathrm{~m} / \mathrm{s}$. The plume diffusion equations for the two cases are given by

$$
\begin{array}{ll}
\text { Category B } & \text { Category E } \\
\sigma_{\mathrm{x}}=\sigma_{\mathrm{y}}=0.299 \mathrm{~d}^{0.9} & \sigma_{\mathrm{x}}=\sigma_{\mathrm{y}}=0.095 \mathrm{~d}^{0.9} \\
\sigma_{\mathrm{z}}=0.04 \mathrm{~d}^{1.2} & \sigma_{\mathrm{z}}=0.41 \mathrm{~d}^{0.58}
\end{array}
$$

where $\mathrm{d}$ is the downwind distance in meters.

If $\mathrm{d}=1 \mathrm{~km}$, then the horizontal diffusion of category $\mathrm{B}$ is approximately 3 times that for category $\mathrm{E}$. The vertical diffusion is, however, a factor of about 210 larger for $\mathrm{B}$ as compared with E. 

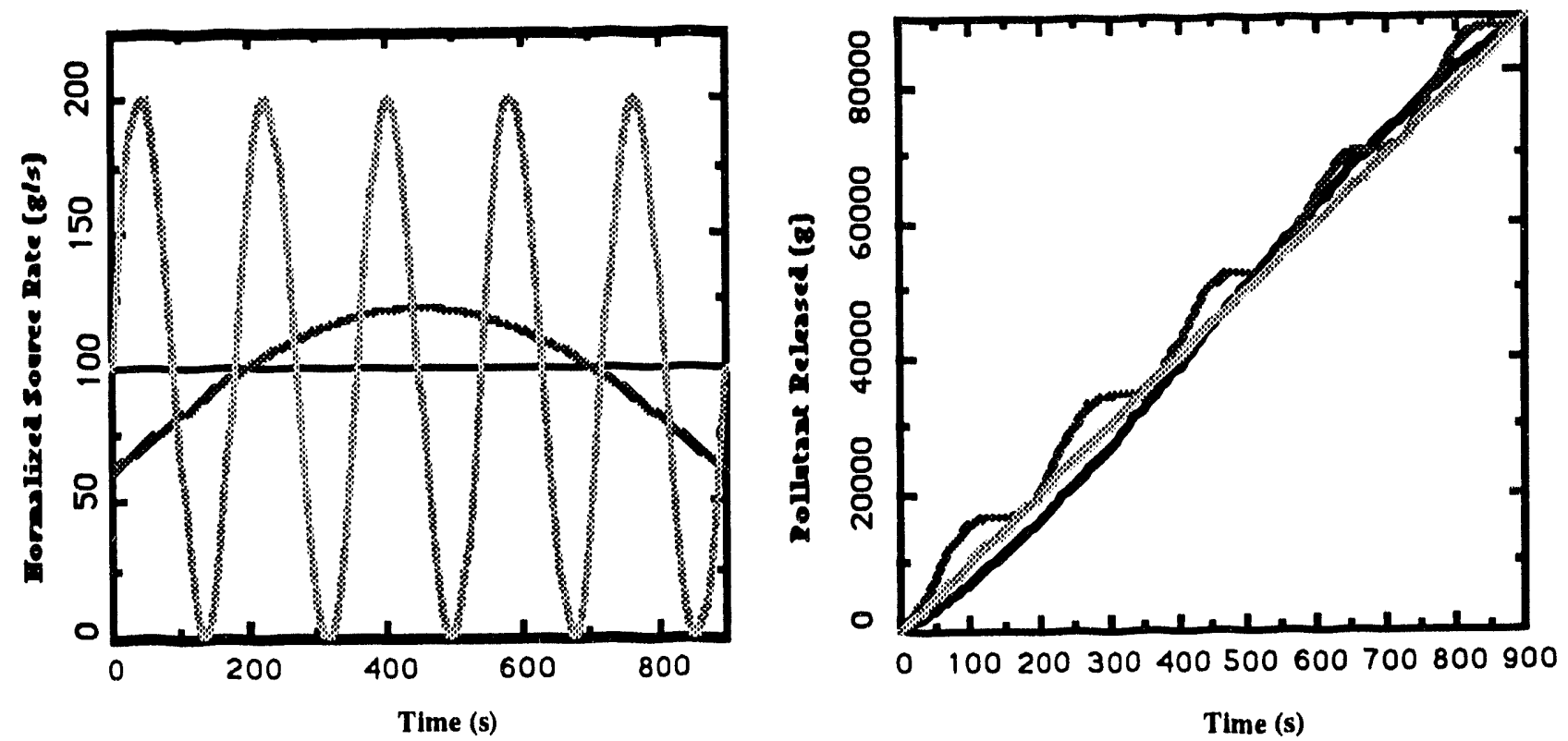

Figure 2: Example source rate function of Eq. 9 with $w=0,1$ and 10 .

Figures 3 (B stability) and 4 (E stability) show the instantaneous rate sampled concentrations at differing downwind distances for the cases of $w=0$, and 10. In Fig. 3 one observes the decay of the sine wave source to around $30 \%$ by $1.0 \mathrm{~km}$ downwind and the total loss of the wave signal at $5.0 \mathrm{~km}$. That is, with a moderately unstable atmosphere, short term wave signals are depleted to undetectable levels in relatively short downwind distances. Figure 4 shows essentially the same diffusion of the signals but at greater downwind distances for the moderately stable atmosphere.

We further note that if the instruments were only accurate to within $30 \%$, then the error bars on both $w=0$ and $w=1$ would overlap to the extent that the two sets of measurements for $w=0$ and $w=10$ would be statistically the same for relatively short downwind distances.

For atmospheric concentration measurements, the typical currently used instruments are unable to measure instantaneous rates as shown in Figures 3 and 4. Rather, these instruments collect the sample over some period of time and report a time-averaged rate. Figure 5 shows the case of $w=10$ with time averaging periods of 1 second and $2,5,10$, and 15 minutes at $1 \mathrm{~km}$ downwind. One can readily see that the time averaging dissipates the peaks and valleys as the averaging time increases. Again, if the instruments have 30\% error margins, then it becomes difficult to separate the source term perturbations from the instrument accuracy with 2 minute and above averaging times even at $1 \mathrm{~km}$ downwind.

As a final note concerning "practical" regularization due to instrumentation and diffusion, at a few $\mathrm{km}$ downwind, using the same 60 samplers, the concentration with $w=100$ in the source term is essentially the same as that with $w=10$. Based on the discussion of this section, for solution of the inverse problem in practical applications, we should not have to be concerned with the ill-posedness of the mathematical form. 

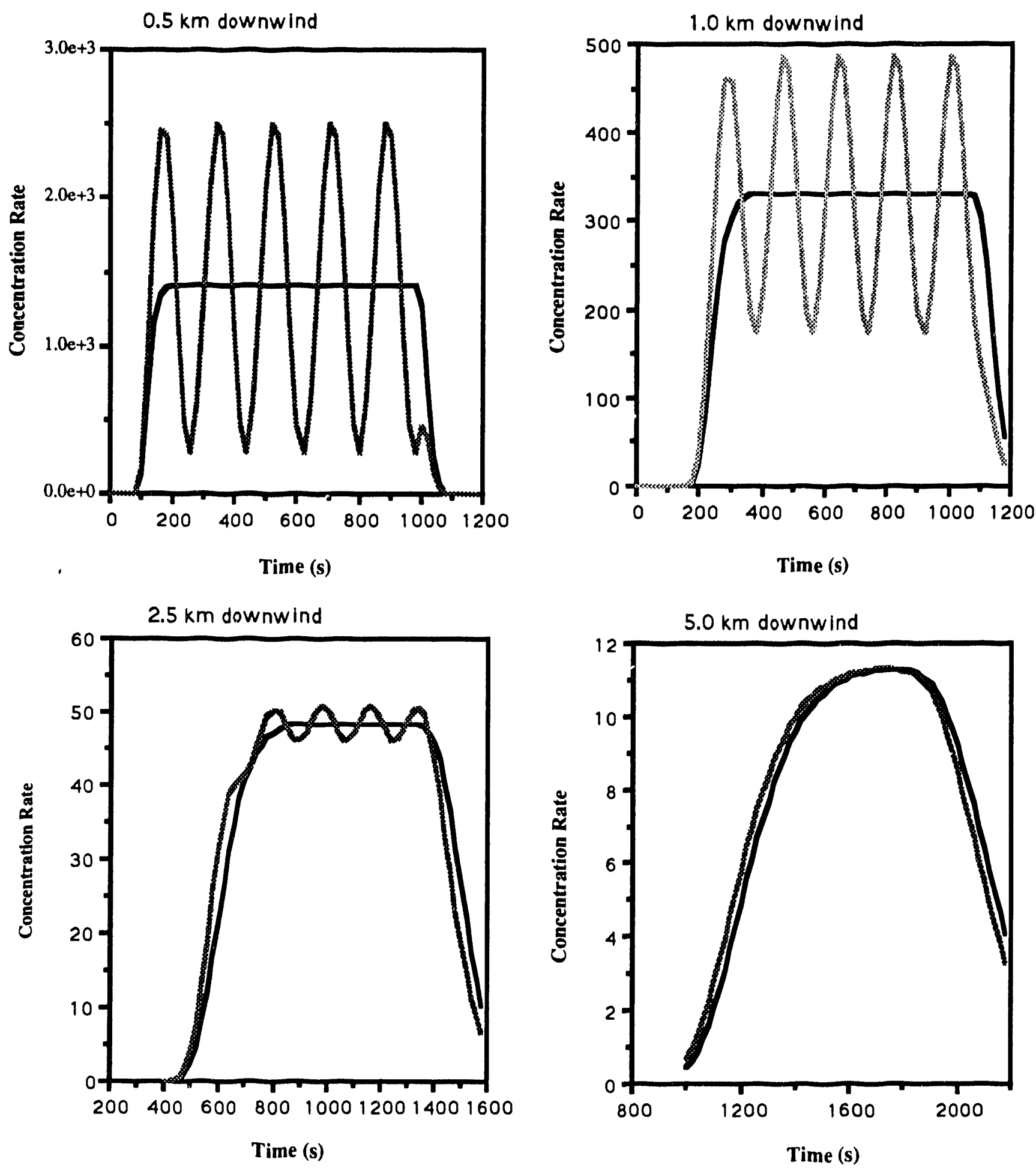

Figure 3: The concentration rate as a function of time for $w=0$ and $w=10$ at differing downwind sampling distances. The stability level is category $B$ with $4 \mathrm{~m} / \mathrm{s}$ average wind speed. 

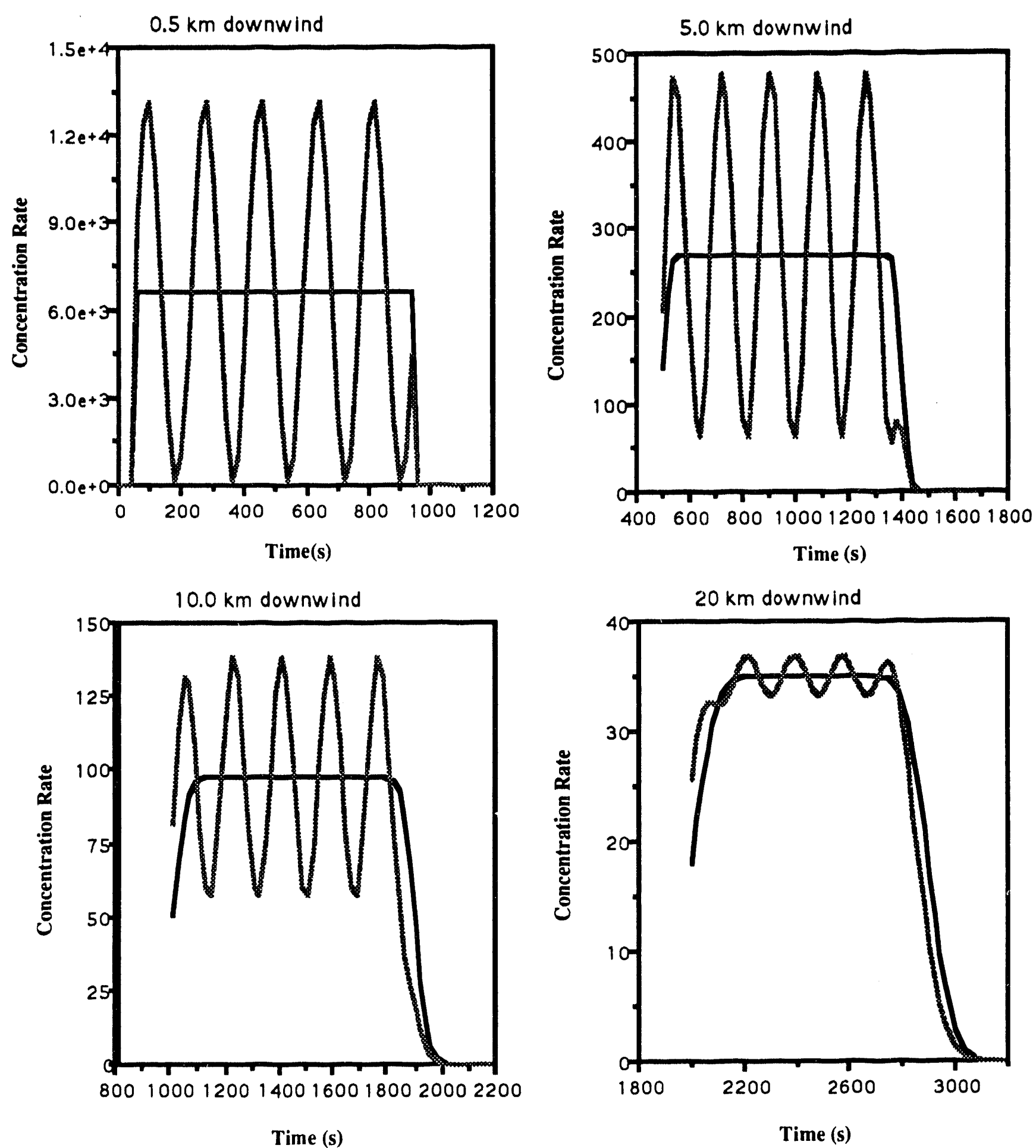

Time (s)

Figure 4: The concentration rate as a function of time for $w=0$ and $w=10$ at differing downwind sampling distances. The stability level is category $E$ with $10 \mathrm{~m} / \mathrm{s}$ average wind speed. 


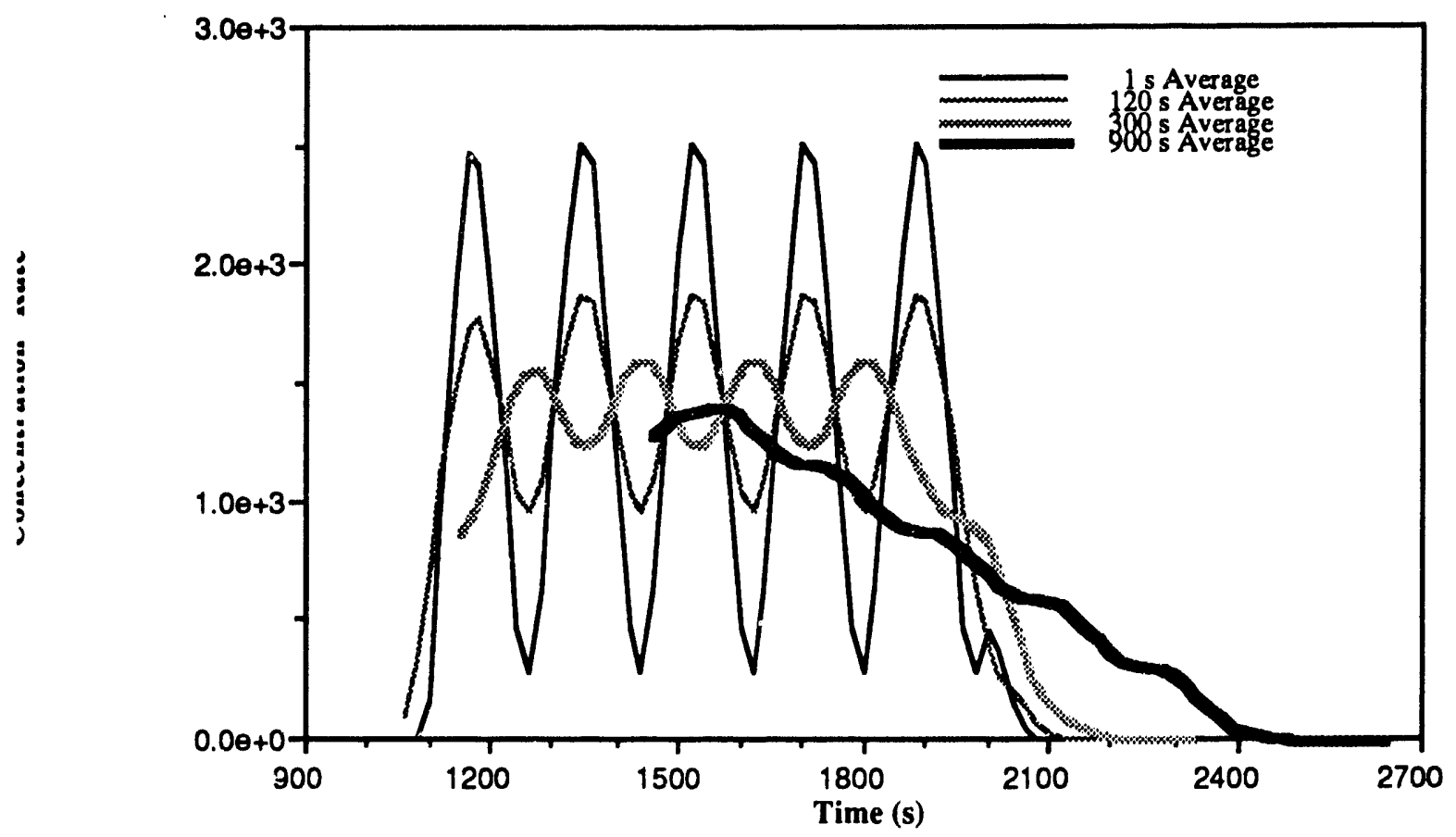

Figure 5: The effects of sampler averaging times at $0.5 \mathrm{~km}$ downwind for $w=10$ in the source rate function. B stability category with average wind speed of $4 \mathrm{~m} / \mathrm{s}$.

\section{The Numerical Algorithms}

\section{Ill-conditionedness of matrices}

In Eq. 7 and 8 we observed that if $\mathbf{A}$ were square and non-singular, then the solution to $\mathbf{A P}=\mathbf{O}$ is given by $\hat{\mathbf{P}}=\mathbf{A}^{-1} \mathbf{O}$. In general, for parameter estimation the number of parameters, $\mathrm{J}$, must be less than or equal to the number of observations, I. For most applications I is larger than J so that $\mathbf{A}$ is not square and the "standard form" (c.f., 18.19.20) for parameter estimation is written

$$
\hat{\mathbf{P}}=\left[\mathbf{A}^{\mathrm{T}} \mathbf{A}\right]^{-1} \mathbf{A}^{\mathrm{T}} \mathbf{O}
$$

where $\mathbf{A}^{\mathrm{T}}$ is the transpose of $\mathbf{A}$.

In previous work (6) we presented algorithms for the solution of Eq. 11 and demonstrated the applicability for estimation of constant rate source strength. We further indicated that for complex source terms, the subject of this work, that we would propose to solve Eq. 11 using the Marquardt (21) methodology of steepest descent coupled with a Taylor's Series expansion. However, in attempting to reproduce complex source terms of the type demonstrated in Section 3 
above, as the averaging times for source rates are increased, the matrix $\mathbf{A}^{\mathrm{T}} \mathbf{A}$ becomes extremely ill-conditionedc and numerically singular.

The numerical algorithms presented in this work approximates a solution to Eq. 7 directly using Householder reflections and Givens rotations (c.f., 22) to minimize the residuals. We implement a slightly modified version of the Non-negative least squares (NNLS) methodology as given by Lawson and Hanson (23).

\section{Algorithms of born-time intervals and implementation}

The first step in source term estimation consists of construction of the "unit response function matrix," F, where we employ the atmospheric transport and diffusion model (SGP in our case for this report). We subdivide the release time of interest into some number, JT, of equal "borntime" intervals. Then the model is executed in such manner such that for a unit release rate at each of the intervals taken individually (all others are set to zero), we compute a response at each of the I observation points, $\left(\mathrm{x}_{\mathrm{i}}, \mathrm{y}_{\mathrm{i}}, \mathrm{z}_{\mathrm{i}}, \mathrm{t}_{\mathrm{i}}\right)$. Thus we produce the I by JT matrix

$$
\mathbf{F}=\left\{\mathrm{f}_{\mathrm{i}, \mathrm{j}}=\operatorname{SGP}\left(\mathrm{x}_{\mathrm{i}}, \mathrm{y}_{\mathrm{i}}, \mathrm{z}_{\mathrm{i}}, \mathrm{t}_{\mathrm{i}}\right) \text { due to unit rateover int erval } \tau_{\mathrm{j}-1} \text { to } \tau_{\mathrm{j}}\right\}
$$

where the model, SGP, may be replaced by any other model, say ADPIC if structured in terms of "born-time" intervals for the tracer particles.

Observe that if we appropriately add columns of F we form the set of "unit response" matrices

$$
\mathbf{F}^{\prime}=\left\{\mathbf{F}_{\varphi} \mid \mathbf{F}_{\varphi} \text { is I by } \varphi, \text { where } \varphi \text { is a divisor of } J T\right\}
$$

which we will use to approximate the source rate by $\mathbf{P}_{\phi}=$ A column vector of $\varphi$ components. Then, consistent with Eq. 6 and Eq. 3, once $\mathbf{P}_{\varphi}$ is known, we compute

${ }^{\mathrm{c}}$ Definition: The condition number, $\operatorname{cond}(K)=\|K\|\left\|K^{-1}\right\|$ where $\|K\|$ denotes a given matricial norm.

It is obvious that $\operatorname{cond}(K)=\|K\|\left\|K^{-1}\right\| \geq\left\|K K^{-1}\right\|=1$. For a linear system, if cond $(K) \approx 1$ the system is called well-conditioned; a system for which $\operatorname{cond}(k) \gg>1$ is call ill-conditioned.

It can be shown that $\operatorname{cond}(K)=\operatorname{cond}\left(K^{-1}\right)$, and for the usual $L_{2}$ norm, we have $\|K\|_{L_{2}} \equiv \sqrt{\max \lambda_{i}\left(K^{*} K\right)}$ where the $\lambda_{i}(A)$ denotes the eigenvalues of the matrix $A$, and $K^{*}$ is the adjoint matrix, from which it can be shown that

$$
\operatorname{cond}(K)=\frac{\sqrt{\max \lambda_{i}\left(K^{*} K\right)}}{\sqrt{\min \lambda_{i}\left(K^{*} K\right)}} .
$$

Observes that cond $\left(\mathrm{K}^{\mathrm{T}} \mathrm{K}\right)=$ cond $(\mathrm{K})^{2}$ so that if $\mathrm{K}$ is ill-conditioned, then $\mathrm{K}^{\mathrm{T}} \mathrm{K}$ is much more ill-conditioned.

Pages 133-138 of Tarantola (20) has a very good numerical example as well as discussion of confidence levels associated with parameter estimations with uncertain data. 


$$
\mathbf{c}_{\varphi}=\mathbf{F}_{\varphi} \mathbf{P}_{\varphi} \text { and } R_{\varphi} \equiv \frac{\sum_{i}\left[\left(\mathrm{o}_{\mathrm{i}}-\mathrm{c}_{\varphi, \mathrm{i}}\right) / \mathrm{o}_{\mathrm{i}}\right]^{2}}{\max \{1, \mathrm{I}-\varphi\}} \text {, }
$$

where $R_{\varphi}$ is the weighted, reduced sum of the squared residuals.

The first step in determination of the parameter vector, $\mathbf{P}_{\varphi}$, is to consider both the row and column vectors of $F_{\varphi}$ in terms of consistencyd. If the $i^{\text {th }}$ row sums to zero and the $i^{\text {th }}$ measurement is non zero, or vice-versa, then both the $i^{\text {th }}$ row and $i^{\text {th }}$ observed values are discarded for parameter estimation. If the remaining $\mathrm{j}^{\text {th }}$ column is entirely zeros, then the $\mathrm{j}^{\text {th }}$ component of $\mathbf{P}_{\varphi}$ cannot be determined by the model and given data. We arbitrarily set this component, $\mathrm{pj}_{\mathrm{j}}$, to zero and remove the column from $\mathrm{F}_{\varphi}$ in formation of the matrix $A_{\varphi}$ given by

$$
A_{\varphi}=\left\{I^{\prime} \leq \mathrm{I} \text { by } \mathrm{J} \leq \varphi \text { from } \mathrm{I}^{\prime}, \mathrm{J} \text { consistant rows, columns of } \mathbf{F}_{\varphi}, \mathrm{J} \leq \mathrm{I}^{\prime}\right\}
$$

and, the NNLS algorithms are used to solve for the J-vector of parameters, $\mathbf{P}_{\varphi}^{\prime}$, as in Eq. 7, i.e.,

$$
\mathbf{A}_{\varphi} \mathbf{P}_{\varphi}^{\prime}=\mathbf{O} \text { subject to } \mathbf{P}_{\varphi}^{\prime} \geq \mathbf{0}
$$

One then combines with the (possibly) previously set values of zero to form the full parameter vector, $\mathbf{P} \varphi$.

\section{Selection of The Minimum Variation Parameter Vector}

From the set of $R_{\varphi}$ computed by Eq. 14 , let $\underline{R}$ be the minimum value over all $\varphi$. If $\underline{R}=R_{\varphi}$ for $\varphi=1$, we select this "constant rate" $p_{1}=\hat{\mathbf{P}}$, the "best estimate" source term. Otherwise, since we generally expect relatively smooth release functions, we choose the vector with the least average variation, $V_{\varphi}$, that has $R_{\varphi}$ within some factor, say 5 , of $R$. That is, if we define

$$
\mathrm{V}_{\varphi}=\frac{\sum_{j=1}^{\varphi-1}\left|p_{\varphi, j+1}-p_{\varphi, j}\right|}{\varphi-1}
$$

we then set $\hat{\mathbf{P}}=\mathbf{P}_{\varphi} \ni R_{\varphi} \leq 5 \underline{R}$ and $V_{\varphi}$ is minimal.

The reason for the minimum variation choice is that, due to random errors associated with sampling instruments, it is possible to find the source rate function that gives the "best fit" in the least squares sense to be more complex than actually warranted by the given data. We will illustrate by example (see Fig. 6) in the following section.

\footnotetext{
d Vector rows are termed inconsistent if either the observed is zero and the computed is non-zero, or, if the observed is non-zero and the computed is zero. Inconsistencies may be caused by wind direction or speed errors, or by atmospheric diffusion or other model parameters which do not adequately represent the physics of the experiment. Clearly, the nigher the number of consistent components, the better our model parameters of wind speed and direction as well as diffusion parameters have captured the essence of the physics of the actual atmospheric conditions during the experiment.
} 


\section{Results of Source Rate Estimations}

In this section we will apply the estimation methodology to several sets of simulated data where the data are generated using the SGP model. A random perturbation of $\pm 30 \%$ will be applied to the model generated data with the restriction that perturbations must leave a nonnegative measured concentration. The first set of calculations will consider the source rate functions of Eq. 9 with $w=0,1$ and 10. The second set of calculations will simulate the possible events of a hypothetical accidental release and demonstrates how the time evolution of events will lead to a reasonable source term estimate.

In all cases we will take $J T=60$, the maximum number of time intervals over the release time. Then, the divisors set, $\Phi$, contains

$$
\Phi=\{\varphi \mid \varphi=1,2,3,4,5,6,10,12,15,20,30,60\} .
$$

\section{Case 1: Simple Test of Methodology}

A total of 90,000 grams of pollutant is released over a period of 15 minutes using the source rate function of Eq. 9. We place 60 samplers at differing down-wind distances. The samplers are assumed to turn on at 20 second intervals and are capable of measuring ground-level concentration rates above $10^{-3} \mu \mathrm{g} / \mathrm{m}^{2} / \mathrm{s}$ to within $30 \%$ accuracy. We assume the samplers can produce either instantaneous ( $1 \mathrm{~s}$ average) rates or time-integrated over 2,5 and 15 minute periods average rates.

To observe the effects of diffusion we also consider two cases: The first case is for B stability category (moderately unstable conditions (4)) and wind speed of $4 \mathrm{~m} / \mathrm{s}$, and a second case for E stability category (slightly stable conditions) with wind speed of $8 \mathrm{~m} / \mathrm{s}$ (although this speed may be somewhat higher than characteristic, the reselts will not be significantly affected).

Tables $1 \mathrm{a}, 1 \mathrm{~b}, 1 \mathrm{c}$, and $1 \mathrm{~d}$ at down-wind distances of $1,1,1.5$ and $5 \mathrm{~km}$, respectively, for $\mathrm{B}$ stability, show the choices of $\varphi$ for the minimum $R_{\varphi}$ and for the minimum average variation, $V_{\varphi}$, choice for $R_{\varphi}$ within a factor of 5 of the minimum $R_{\varphi}$.

One observes, with the exception of $120 \mathrm{~s}$ average ${ }^{e}$ for $w=10$ at $0.5 \mathrm{~km}$, the minimum variation choice reduces the value of $\varphi$, the number of step intervals in the final estimation choice. It is also a general pattern that as the samplers are moved further from the source that $\varphi$ decreases, and similarly as the sample averaging time is increased. In fact, for this case, for 2.5 and $5 \mathrm{~km}$ downwind, the minimum variation source estimation is just a constant rate with a single exception (again caused by only a slight overshoot of the factor of 5 on an extremely small $R$. For a single interval in this case, $R=1.428$ which is a highly acceptable fit). One would expect this to happen based on Figures 3,4 and 5 of the preceding section.

The last two columns compare the total released mass using the integral of the appropriate step functions. Note that for $\mathrm{R}_{\min }$ the minimum value is 87016 grams, about $3.3 \%$ low, whereas the maximum value is 95546 grams, about $6.2 \%$ high. The $V_{\text {min }}$ values are $89008,1.1 \%$ low and $94545,5 \%$ high, a slightly better fit than for the $R_{\min }$. In either case, this is certainly an

\footnotetext{
e For $\varphi<10 \mathrm{R}$ ranges from 89. to 129., more than a factor of 5 Isrger than the minimum. The remaining sets of intervals have $R \leq 14$. For example, with 20 intervals, $R=9.86$, only trivially larger than the chosen minimum, so we are not alarmed by this apparent anomoly.
} 
excellent estimation of the total mass released in view of the fact that we allow random $30 \%$ errors on the "observed" data.

Table 1a: Samplers are $0.5 \mathrm{~km}$ downwind with stability category $\mathrm{B}$.

\begin{tabular}{|c|c|c|c|c|c|c|c|c|c|}
\hline \multirow[t]{2}{*}{ w } & \multirow{2}{*}{$\begin{array}{c}\text { Average } \\
\text { (sec) }\end{array}$} & \multicolumn{2}{|c|}{$\varphi$ at } & \multicolumn{2}{|c|}{$R_{\varphi}$ at } & \multicolumn{2}{|c|}{$V_{\varphi}$ at } & \multicolumn{2}{|c|}{ Total Released (g) } \\
\hline & & $R_{\min }$ & $V_{\min }$ & $R_{\min }$ & $v_{\min }$ & $R_{\min }$ & $V_{\min }$ & $R_{\min }$ & $V_{\text {min }}$ \\
\hline 0 & & 30 & 1 & 7.02 & 9.50 & 28.08 & 0.00 & 92334 & 92247 \\
\hline 1 & $\overline{1}$ & 30 & $\overline{12}$ & 5.86 & 11.77 & 31.07 & 9.92 & 89921 & 90073 \\
\hline 10 & 1 & 30 & 30 & 16.33 & 16.33 & 66.57 & 66.57 & 91023 & $9 \overline{1023}$ \\
\hline$\overline{0}$ & 120 & 4 & 1 & 5.91 & 6.72 & 2.82 & 0.00 & 89834 & 89955 \\
\hline$\frac{1}{1}$ & 120 & 20 & 6 & 4.16 & 8.23 & 106.37 & 17.03 & 91610 & 91902 \\
\hline 10 & 120 & 10 & 20 & 9.11 & 9.86 & 121.69 & 84.61 & 89172 & 89334 \\
\hline 0 & 300 & 2 & 1 & 10.13 & 10.99 & 3.76 & 0.00 & 89201 & 89275 \\
\hline$\overline{1}$ & 300 & 15 & 1 & 9.04 & 32.13 & 74.6 & 0.00 & 86226 & 91068 \\
\hline 10 & 300 & 10 & 5 & 8.16 & 37.13 & 170.94 & 16.82 & 91162 & 90219 \\
\hline 0 & 900 & 5 & 1 & 3.77 & 4.59 & 32.00 & 0.00 & 95546 & 91882 \\
\hline 1 & 900 & 4 & 1 & 4.99 & 9.41 & 22.33 & 0.00 & $9 \longdiv { 1 4 1 8 }$ & 92312 \\
\hline 10 & 900 & 20 & $\overline{1}$ & 5.24 & $\overline{16.91}$ & 133.70 & 0.00 & 91969 & $\overline{89901}$ \\
\hline
\end{tabular}

Table 1b: Samplers are $1 \mathrm{~km}$ downwind with stability category $\mathrm{B}$.

\begin{tabular}{|c|c|c|c|c|c|c|c|c|c|}
\hline \multirow[t]{2}{*}{ w } & \multirow{2}{*}{$\begin{array}{c}\text { Average } \\
\text { (sec) }\end{array}$} & \multicolumn{2}{|c|}{$\varphi$ at } & \multicolumn{2}{|c|}{$\overline{R_{\varphi} \text { at }}$} & \multicolumn{2}{|c|}{$V_{\varphi}$ at } & \multicolumn{2}{|c|}{ Total Released (g) } \\
\hline & & $\mathbf{R}_{\min }$ & $V_{\min }$ & $R_{\min }$ & $v_{\min }$ & $R_{\min }$ & $V_{\min }$ & $R_{\min }$ & $V_{\min }$ \\
\hline 0 & 1 & 20 & 1 & 2.36 & 2.79 & 48.70 & 0.00 & 90608 & 90645 \\
\hline$\frac{1}{1}$ & 1 & 12 & 12 & 2.64 & 2.64 & 11.56 & 11.56 & 89809 & 89809 \\
\hline 10 & 1 & 20 & 20 & 2.98 & 2.98 & 90.24 & 90.24 & 92125 & 92125 \\
\hline 0 & 120 & 6 & 1 & 1.82 & 1.95 & 4.59 & 0.00 & 89848 & 89802 \\
\hline$\frac{\pi}{1}$ & 120 & 20 & $\frac{6}{6}$ & 0.91 & 2.06 & 154.90 & 15.64 & $91+67$ & 91467 \\
\hline 10 & 120 & 20 & 20 & 1.81 & 1.81 & 123.35 & $12 j .35$ & $\overline{89481}$ & 89481 \\
\hline$\overline{0}$ & 300 & 15 & 1 & 2.30 & 2.59 & 73.16 & 0.00 & 89371 & 89808 \\
\hline 1 & 300 & 10 & 1 & 2.06 & 8.88 & 79.37 & 0.00 & 87843 & 90583 \\
\hline 10 & 300 & 10 & 1 & 1.75 & 8.51 & 94.58 & 0.00 & 89767 & 89759 \\
\hline 0 & 900 & 3 & 1 & 1.07 & $1 . \overline{18}$ & 8.97 & 0.00 & 92590 & 90986 \\
\hline 1 & 900 & 4 & 1 & 1.32 & 2.33 & 19.36 & 0.00 & 90897 & 92691 \\
\hline 10 & 900 & 12 & $\overline{1}$ & 1.69 & 3.4 & 110.43 & 0.00 & 90778 & 90747 \\
\hline
\end{tabular}

From the Tables $1 \mathrm{a}, 1 \mathrm{~b}, 1 \mathrm{c}$, and $1 \mathrm{~d}$ we observe that for the constant release, $w=0$, the $V_{\text {min }}$ parameter estimations are done with 4 or fewer intervals over all of the downwind distances and sampling averaging times. From the model produced data, the estimated parameters range from a minimum of 88 to a maximum of 108 , excellent estimations in view of the $30 \%$ accuracy of the samplers. We shall not further pursue the constant rate release case in this work.

Table 2 is for the E stability case. Here we show the estimations based on the instantaneous rate only. Note that the downwind distances are somewhat greater than for the B stability case. We note that in general, with the smaller diffusion of the E stability, the source term is reconstructed with more variability at downwind distances. Again, the total released mass is within a few percent of the actual release. 
Table 1c: Samplers are $2.5 \mathrm{~km}$ downwind with stability category $\mathrm{B}$.

\begin{tabular}{|c|c|c|c|c|c|c|c|c|c|}
\hline \multirow[t]{2}{*}{$w$} & $\begin{array}{l}\text { Average } \\
(\mathrm{sec})\end{array}$ & \multicolumn{2}{|c|}{$\stackrel{\varphi \text { at }}{\mathrm{R}_{\mathrm{min}}}$} & \multicolumn{2}{|c|}{$\mathrm{R}_{\varphi} \underset{V_{m}}{\mathrm{al}}$} & \multicolumn{2}{|c|}{$v_{\varphi} a t$} & \multicolumn{2}{|c|}{$\begin{array}{l}\text { Total Released (g) } \\
\mathbf{R}_{\min } \mathrm{V}_{\min }\end{array}$} \\
\hline & 1 & 2 & $\frac{11}{1}$ & $3.17 \mathrm{e}-1$ & $3.29 \mathrm{e}-1$ & 1.84 & 0.00 & 91197 & 91200 \\
\hline 1 & 1 & 10 & 1 & $3.10 \mathrm{e}-1$ & 1.43 & 41.21 & 0.00 & 89662 & 92297 \\
\hline 10 & 1 & 10 & $\overline{1}$ & $3.09 \mathrm{e}-1$ & 1.01 & $171.8^{\circ}$ & 0.00 & 92150 & 92219 \\
\hline 0 & 120 & 3 & 1 & $2.34 \mathrm{e}-1$ & $2.56 \mathrm{e}-1$ & 3.55 & 0.00 & 89571 & 89769 \\
\hline 1 & 120 & $\overline{12}$ & $\overline{6}$ & $1.76 \mathrm{e}-1$ & $2.48 \mathrm{e}-1$ & 109.33 & 17.97 & 91383 & 91983 \\
\hline 10 & 120 & $\overline{10}$ & 1 & $2.61 \mathrm{e}-1$ & 1.08 & 74.12 & 0.00 & 89275 & 89008 \\
\hline 0 & 300 & 4 & 1 & $3.43 \mathrm{e}-1$ & $3.68 \mathrm{e}-1$ & 8.87 & 0.00 & 89693 & $8 \overline{9733}$ \\
\hline 1 & 300 & 10 & 1 & $3.24 \mathrm{e}-2$ & $9.98 \mathrm{e}-1$ & 152.35 & 0.00 & 87221 & 90838 \\
\hline 10 & 300 & 6 & 1 & $2.63 \mathrm{c}-1$ & $7.55 \mathrm{e}-1$ & 57.19 & 0.00 & 89870 & 89899 \\
\hline 0 & 900 & 6 & 1 & $1.46 \mathrm{e}-1$ & $1.66 \mathrm{e}-1$ & 118.89 & 0.00 & 94196 & 91184 \\
\hline$\pi$ & 900 & 4 & 1 & $1.78 \mathrm{e}-1$ & $2.95 \mathrm{e}-2$ & 21.98 & 0.00 & 90977 & 92607 \\
\hline 10 & 900 & 4 & 1 & $2.01 \mathrm{e}-1$ & $3.77 \mathrm{e}-1$ & 14.47 & 0.00 & 90302 & 90559 \\
\hline
\end{tabular}

Table 1d: Samplers are $5 \mathrm{~km}$ downwind with stability category B.

\begin{tabular}{|c|c|c|c|c|c|c|c|c|c|}
\hline \multirow[t]{2}{*}{$w$} & \multirow{2}{*}{$\begin{array}{l}\text { Average } \\
\text { (sec) }\end{array}$} & \multicolumn{2}{|c|}{$R_{\min }^{\varphi \text { at }} V_{\min }$} & \multicolumn{2}{|c|}{$\mathrm{R}_{\min }^{\mathrm{R}} \stackrel{\mathrm{V}}{\mathrm{a}}_{\mathrm{min}}$} & \multicolumn{2}{|c|}{$\mathrm{V}_{\min }^{\mathrm{V}_{\mathrm{V}}}$} & \multirow{2}{*}{\multicolumn{2}{|c|}{$\begin{array}{l}\text { Total Released (g) } \\
R_{\min } V_{\min }\end{array}$}} \\
\hline & & $\frac{\pi n}{2}$ & min & $\frac{R_{\text {min }}}{726 e-2}$ & $\frac{V_{\min }}{7.54 \mathrm{e}-2}$ & $\frac{\pi \operatorname{mon}}{2.22}$ & $\frac{v_{\min }}{0.00}$ & & \\
\hline 1 & & $\frac{\pi}{4}$ & 1 & $7.08 \mathrm{e}-2$ & $1.98 \mathrm{e}-1$ & 24.78 & 0.00 & 90015 & 93013 \\
\hline 10 & 1 & 6 & $\overline{1}$ & $7.37 \mathrm{e}-2$ & $1.14 \mathrm{e}-1$ & 38.43 & 0.00 & 92137 & 92238 \\
\hline 0 & 120 & 3 & 1 & $5.41 \mathrm{e}-2$ & $5.78 \mathrm{e}-2$ & 4.67 & 0.00 & 89498 & 89865 \\
\hline 1 & 120 & 6 & 1 & $4.73 e-2$ & $1.99 \mathrm{c}-1$ & 20.03 & 0.00 & 91374 & 94545 \\
\hline 10 & 120 & 5 & 1 & $5.91 \mathrm{e}-2$ & $1.45 \mathrm{e}-1$ & 43.73 & 0.00 & 89574 & 89095 \\
\hline 0 & 300 & 4 & 1 & $8.38 \mathrm{e}-2$ & $8.40 \mathrm{c}-2$ & 22.48 & 0.00 & 89602 & 89755 \\
\hline 7 & 300 & 6 & 1 & $7.91 \mathrm{c}-2$ & $1.48 \mathrm{e}-1$ & 109.51 & 0.00 & 87016 & 90772 \\
\hline 10 & 300 & 4 & 1 & $5.62 \mathrm{e}-2$ & $1.20 \mathrm{e}-1$ & 42.64 & 0.00 & 89674 & 89928 \\
\hline 0 & 900 & 3 & 1 & $3.49 \mathrm{e}-2$ & $3.94 \mathrm{e}-2$ & 22.71 & 0.00 & 93866 & 91040 \\
\hline 1 & 900 & 3 & 1 & $4.14 \mathrm{e}-2$ & $5.60 \mathrm{e}-2$ & 29.86 & 0.00 & 90999 & 92492 \\
\hline 10 & $9(x)$ & 3 & 7 & $4.66 \mathrm{e}-2$ & $7.76 c-2$ & 31.95 & 0.00 & 89808 & $9(1) 642$ \\
\hline
\end{tabular}

Table 2: Stability category $E$ with 1 second averaging at differing downwind distances..

\begin{tabular}{|c|c|c|c|c|c|c|c|c|c|}
\hline \multirow[t]{2}{*}{$w$} & \multirow{2}{*}{$\begin{array}{c}\text { Distance } \\
(\mathrm{km})\end{array}$} & \multicolumn{2}{|c|}{$\varphi$ at } & \multicolumn{2}{|c|}{$\mathrm{R}_{\varphi}$ at } & \multicolumn{2}{|c|}{$V_{\varphi}$ at } & \multicolumn{2}{|c|}{ Total Released (g) } \\
\hline & & $R_{\min }$ & $V_{\min }$ & $R_{\min }$ & $v_{\text {min }}$ & $R_{\min }$ & $V_{\min }$ & $R_{\min }$ & $V_{\text {min }}$ \\
\hline 0 & 0.5 & 15 & 1 & 48.70 & 50.26 & 6.36 & 0.00 & 92589 & 92589 \\
\hline 1 & 0.5 & 12 & 20 & 35.15 & 44.15 & 10.81 & 7.66 & 90318 & 90241 \\
\hline 10 & 0.5 & 30 & 30 & 80.36 & 80.36 & 63.30 & 63.30 & 91020 & 91020 \\
\hline 0 & 1.0 & 12 & 1 & 19.16 & 19.87 & 5.40 & 0.00 & 92568 & 92502 \\
\hline 1 & 1.0 & 15 & 20 & 16.51 & 22.60 & 9.64 & 7.20 & 89968 & 89977 \\
\hline 10 & 1.0 & 30) & 30 & 152.32 & 152.32 & 64.43 & 64.43 & 88853 & 88853 \\
\hline 0 & 10.0 & 15 & 1 & 0.61 & 0.66 & 26.10 & 0.00 & 92052 & 92112 \\
\hline 1 & 10.0 & 12 & 10 & 0.44 & 0.44 & 11.90 & 11.72 & $9(1683$ & 90676 \\
\hline 10 & 10.0 & 20 & 20 & 0.90 & 0.90 & 92.20 & 92.20 & 89933 & 89933 \\
\hline 0 & 20.0 & 10 & 1 & 0.21 & 0.21 & 25.68 & $0.0(x)$ & 91539 & 92253 \\
\hline 1 & 20.0 & 6 & 6 & 0.13 & 0.13 & 17.22 & 17.22 & 90788 & $9(0) 788$ \\
\hline 10 & 20.0 & 10 & 1 & 0.27 & 0.94 & 152.45 & 0.00 & 89339 & 89171 \\
\hline
\end{tabular}



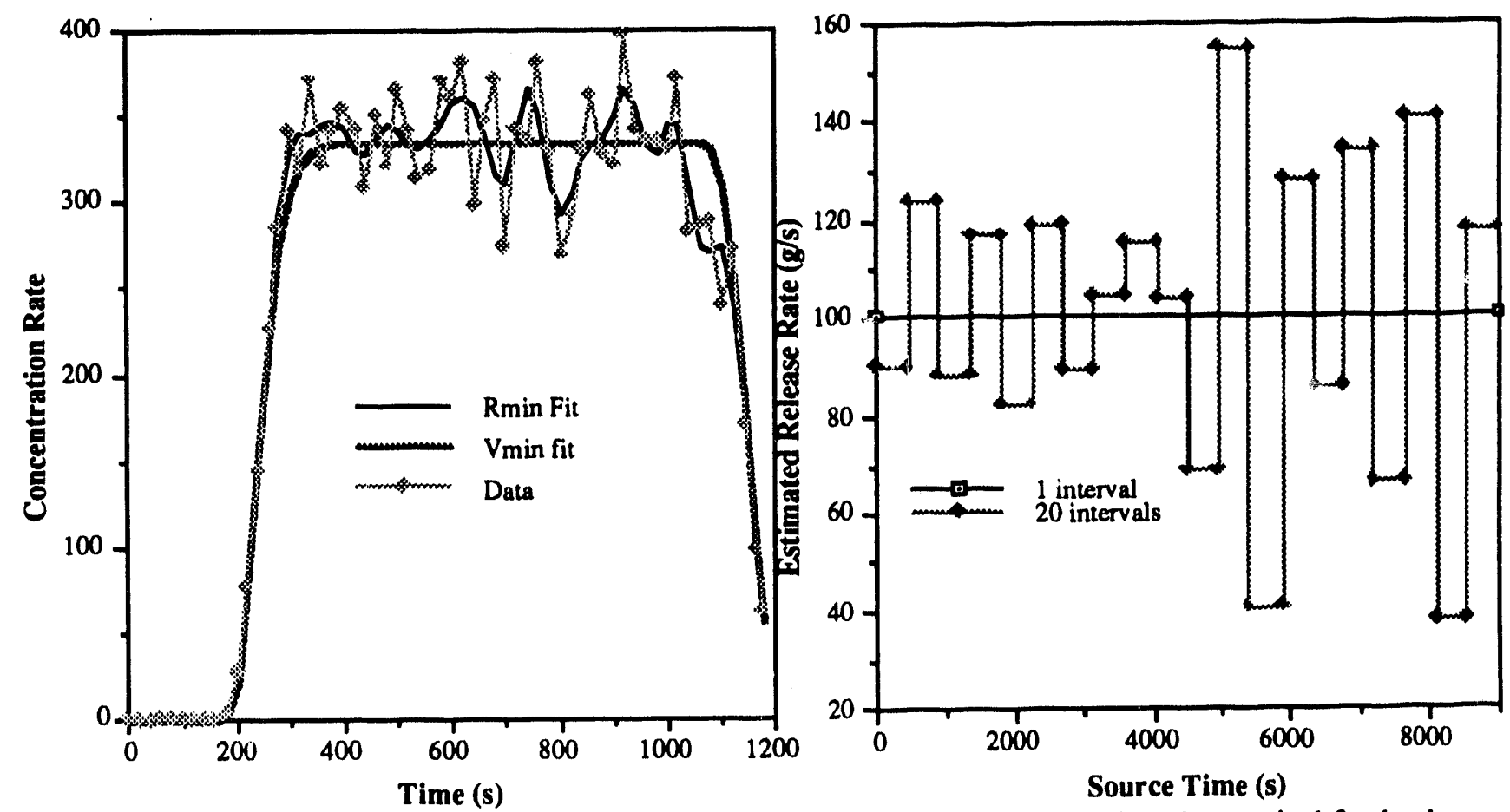

Figure 6: Comparisons of the estimated surce rate and the fit to the data $1 \mathrm{~km}$ downwind for both the $R$ min and Vmin parameter sets for $w=0$ and $1 \mathrm{~s}$ averaging on samplers.

Figure 6 shows results for a constant release rate $(w=0)$ when the samplers are situated $1 \mathrm{~km}$ downwind and use a $1 \mathrm{~s}$ time averaging for the rates. From Table $1 \mathrm{a}$ we observe that the $\mathbf{R}_{\mathrm{min}}$ estimation uses 20 intervals with $R_{20}=2.36, V_{20}=48.7$ and total released mass that is $0.68 \%$ high. The $V_{\text {min }}$ estimation uses intervals with $R_{1}=2.79, V_{1}=0.0$ and total released mass $0.72 \%$ high. Observe that the $R_{\text {min }}$ estimation has interval values ranging from 38 to 155 whereas the $V_{\text {min }}$ estimation is 100.72 , almost exactly the actual value of $100 \mathrm{~g} / \mathrm{s}$. Figure 6 also compares the estimated fits to the data. The data has random perturbations of $\pm 30 \%$ about the smooth values generated by SGP. Observe that the $R_{\min }$ fit attempts to follow the data variations whereas the $V_{\text {min }}$ estimation is essentially smooth, clearly a better fit to "reality" if our samplers were perfect.

Figures 7 shows the actual and estimated $\left(V_{\min }\right)$ release functions for the cases of $w=1$ and $w=10$ again with the $30 \%$ accuracy of samplers. The figures overlay the estimates made for both the $B$ and $E$ stability categories. At this short distance the diffusion effects are quite small and the instantaneous (1 s average) sampling reproduces the actual source to within about the $30 \%$ perturbations. We observe that by $5 \mathrm{~km}$ downwind the B category diffusion has lost all of the perturbations whereas the E category still allows a good source function reconstruction. 


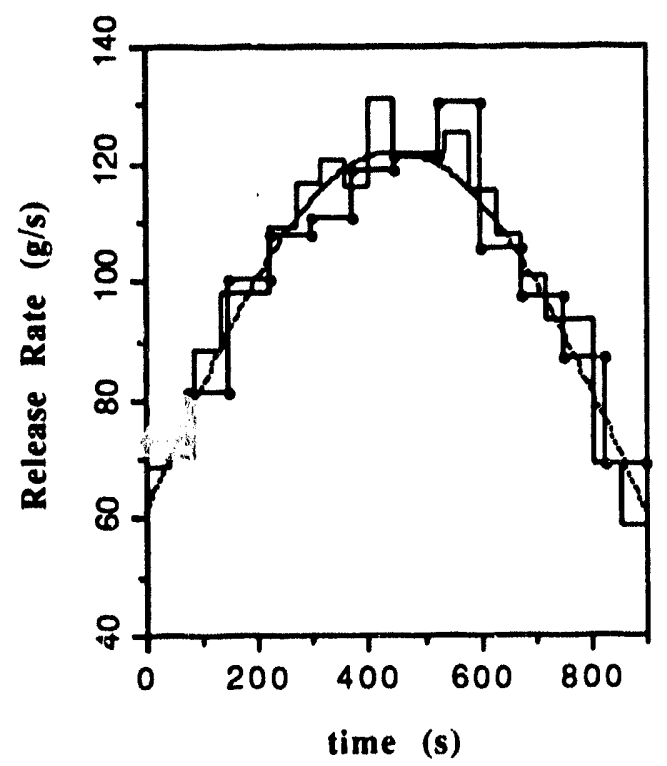

Figure 7a: $0.5 \mathrm{~km}$ downwind, $w=1$.

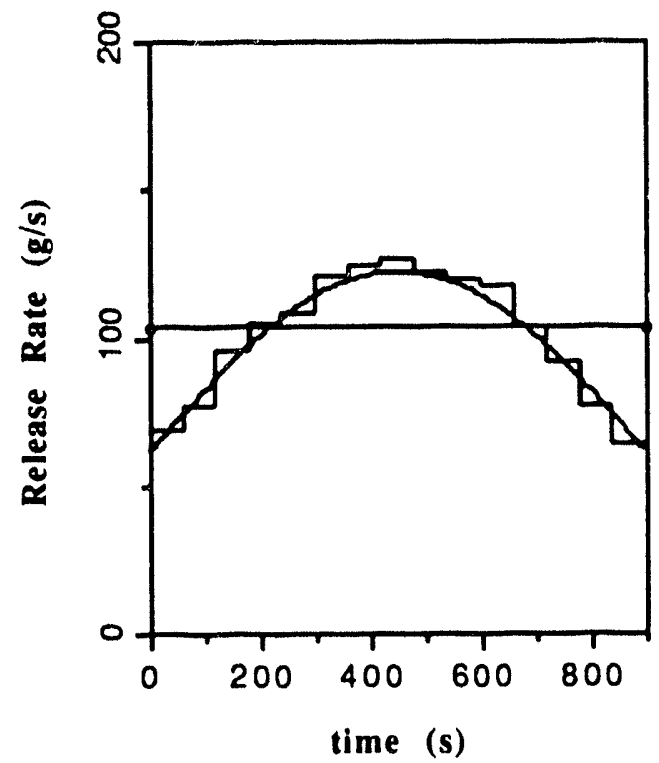

Figure 7c: $5.0 \mathrm{~km}$ downwind, $w=1$.

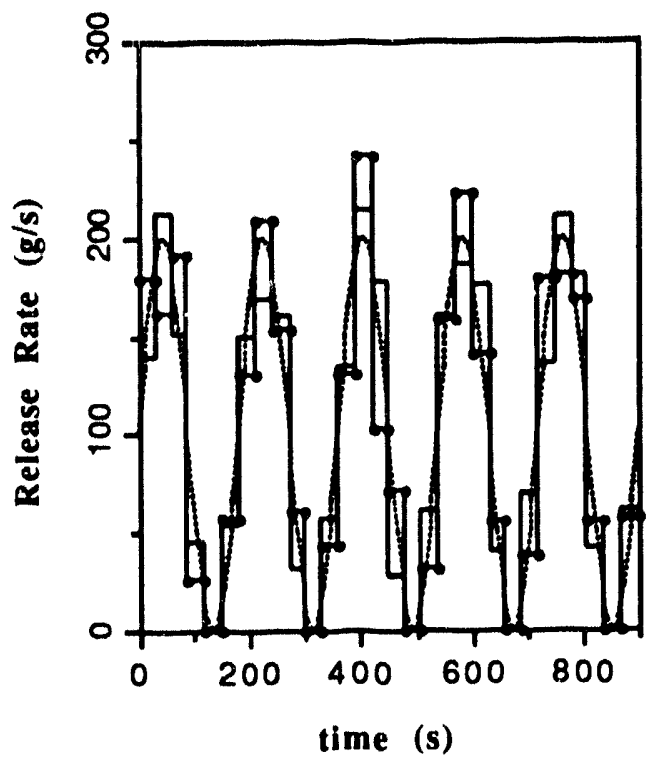

Figure 7b: $0.5 \mathrm{~km}$ downwind, $\mathrm{w}=10$.

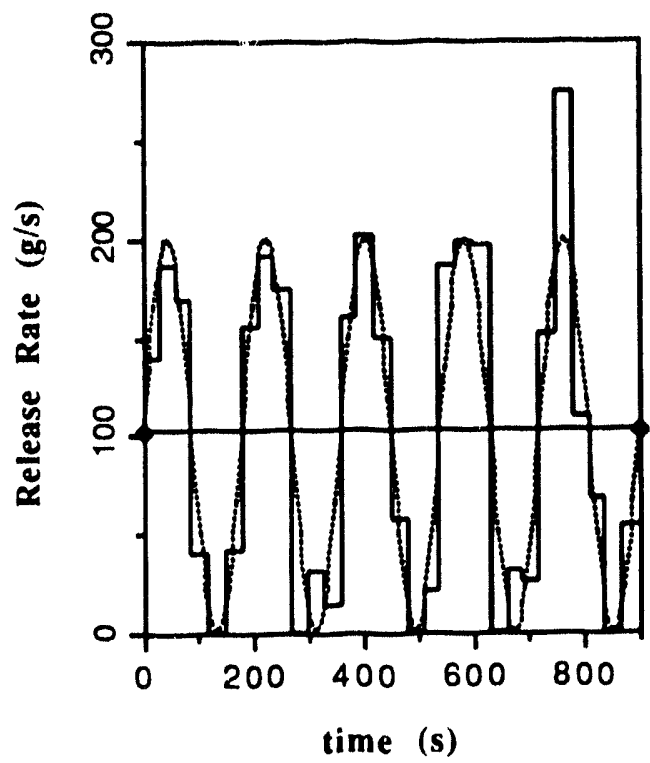

Figure 7d: $5.0 \mathrm{~km}$ downwind, $w=10$.

Figure 7: Estimated source functions for $w=1$ and $w=10$ using instantaneous sampling measurements at 0.5 and $5.0 \mathrm{~km}$ downwind. The dotted line is the actual release function, the step function with symbol for B stability, and the plain line step function for E stability. 


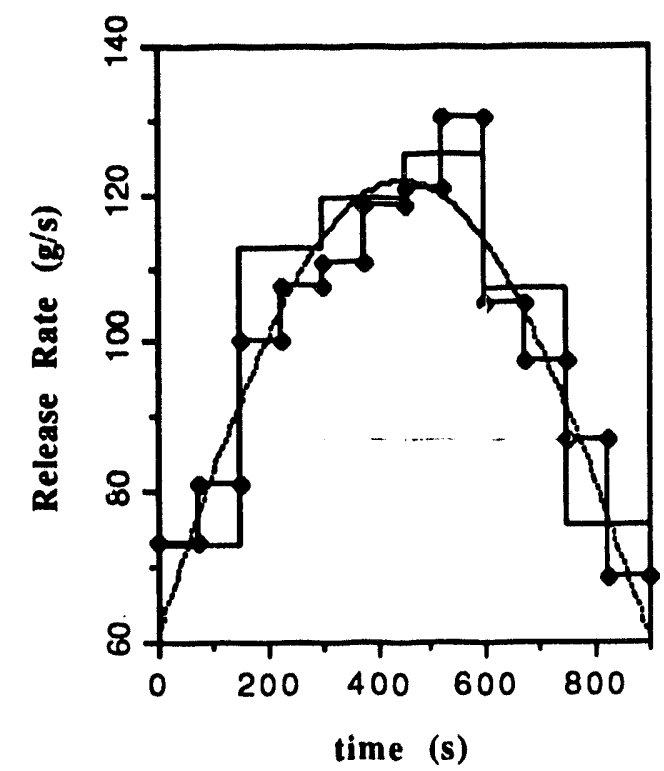

Figure 8a: Average times $=1,120 \mathrm{~s}, \mathrm{w}=1$.

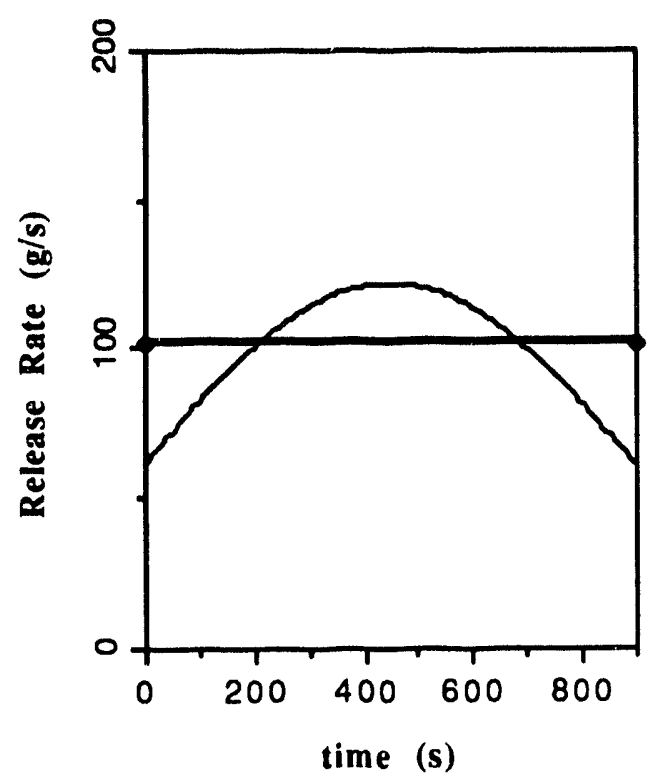

Figure 8c: Average times=300, $900 \mathrm{~s}, w=1 . \quad$ Figure 8d: Average times $=300,900 \mathrm{~s}, \mathrm{w}=10$.

Figure 8: Estimated source functions for $w=1$ and $w=10$ for $B$ stability at $0.5 \mathrm{~km}$ downwind.. The dotted line is the actual release function, the step function with symbol for the shorter indicated averaging time and the plain line for the longer averaging time. 
Figure 8 shows the effects of sampler averaging time on the estimated source functions. The perturbations are averaged out with increasing samplers averaging times increase just as one would expect from Figures 3,4 and 5.

This set of test cases shows the accuracy of estimation that one might expect from the methodology. Obviously the accuracy of the numerics depends on the accuracy of the observed data used for the estimations, and as we shall see in the following example, how well we have reproduced the atmospheric transport and diffusion processes with our numerical model.

\section{Case 2: A Simulated Emergency Response Source Term Estimation}

Since the real purpose of this paper is to show a method of source term estimation that may be used by decision makers in actual cases of accidental release of toxics to the atmosphere, we simulate ${ }^{f}$ such a situation. The data are again model generated with $30 \%$ random perturbations using the source term shown in Figure 9. The release duration is 2.5 hours and consists of an "explosion" followed by a slow build-up, a second explosion, then "shut-down." A total of $1.15 \times 10^{6}$ grams of gaseous pollutant is released. The release occurs with slightly unstable atmospheric conditions (stability category $C$ ) and average wind speed of $6 \mathrm{~m} / \mathrm{s}$.

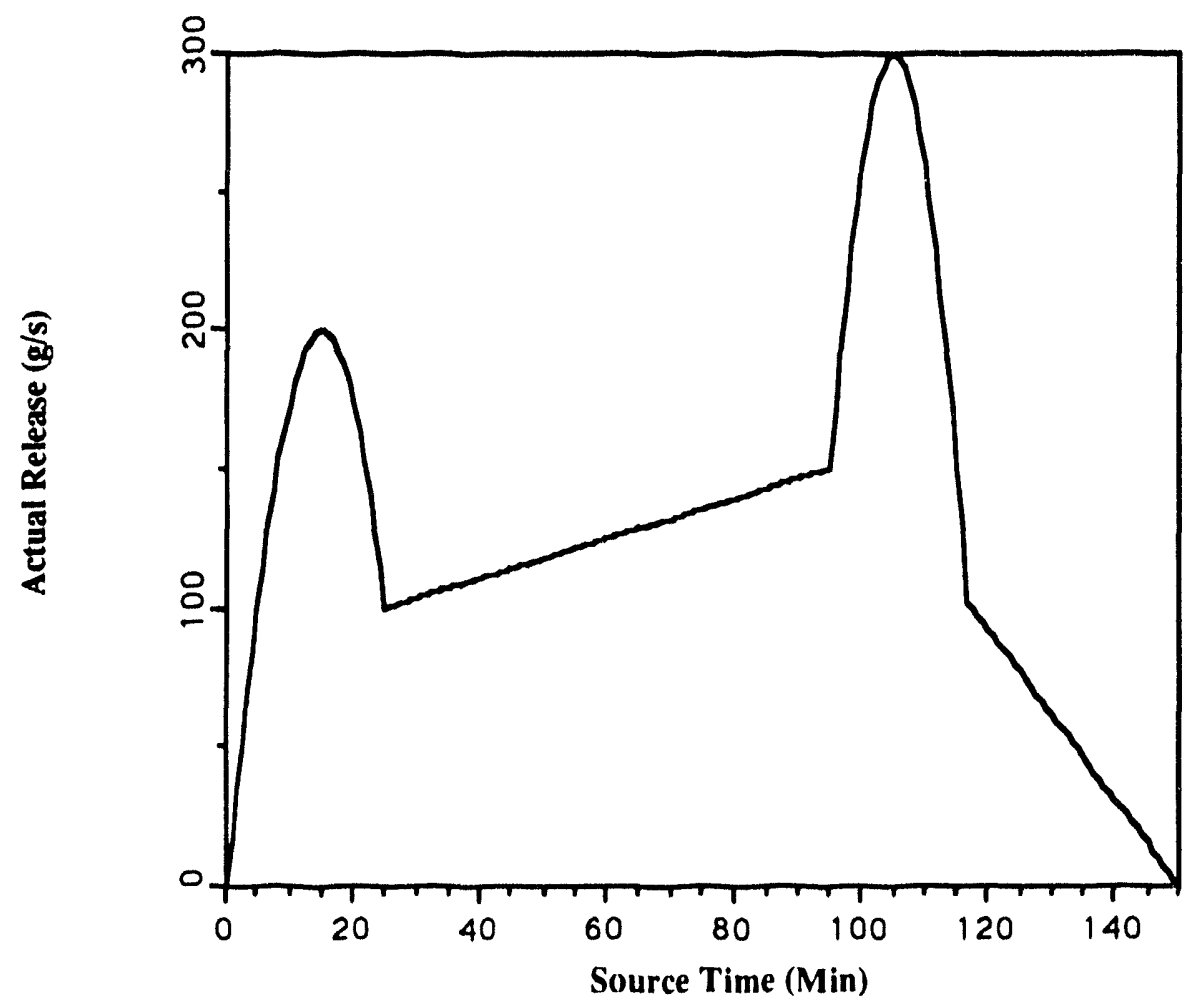

Figure 9: The actual release of pollutant at the source of the simulated accident.

$f$ The primary reasons for using model generated data are:

1) Real accidents with complex releases and well-measured data, including the complete atmospheric conditions are rare.

2) We wish to be able to separate out the effects of data uncertainties.

3) We wish to be able to comment on how well we do numerically with the actual situation. 
We assume there are 2 samplers to provide the dara. The samplers are located at 0.5 and 1.0 $\mathrm{km}$ downwind from the source. The samplers operate continuously in time and provide timeaveraged concentration rates once each 5 minutes in real time. The "real" and measured data concentration rates are shown in Figure 10.

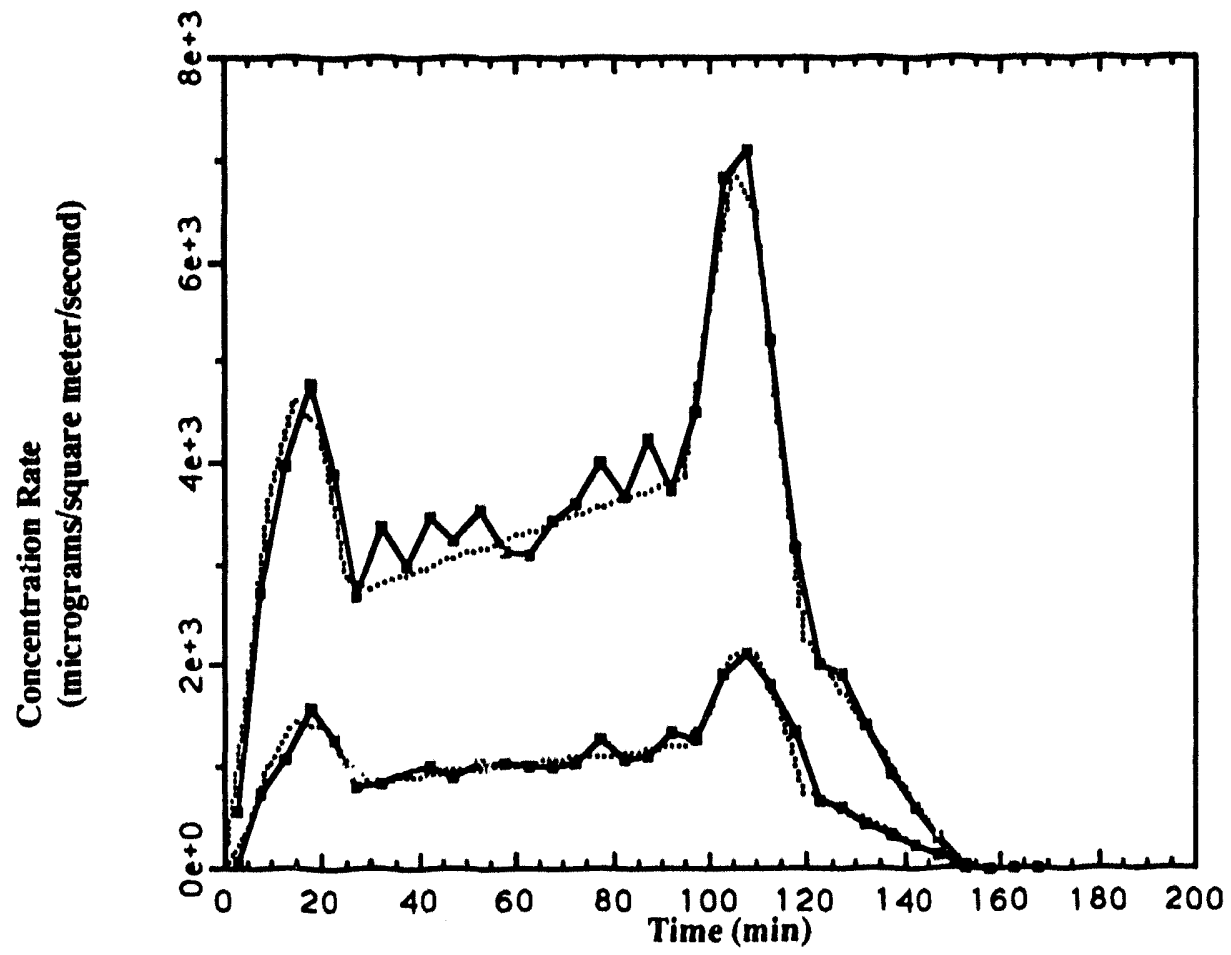

Figure 10: The simulated data (with plot symbols) for 5 minute average rates at 0.5 and $1.0 \mathrm{~km}$ downwind. The actual concen'ration rates are shown with dotted lines.

The data are assumed available to the emergency response assessors in real time. That is, at each 5 minutes since accident start, we receive 2 data points, one from the sampler at $0.5 \mathrm{~km}$ downwind and a second from the sampler at $1.0 \mathrm{~km}$ downwind. The source estimation code uses all data available at a given time to reconstruct the source rate and estimate the concentration rates at 10, 20 and $30 \mathrm{~km}$ downwind. The estimated concentrations would, in turn, be used by the decision makers to determine what, if any, protective procedures to implement.

Figures $11 \mathrm{a}$ through $11 \mathrm{j}$ show the estimated source term and the predicted (based on the estimated scurce) concentration rates as functions of real time since the start of the accident. The predicted concentration rates are for 10,20 and $30 \mathrm{~km}$ downwind. The figures start with 5 minute increments of time and then for 30 minute increments. The source estimate computes the rate for as many increments as the measurements warrant (e.g., 2 measurements means up to 2 intervals, 4 measurements means up to 4 intervals, etc.) and the remainder of the source duration time is filled with the value of the last computed non-zero rate. For this example we have chosen to set the estimated source end time initially to 10000 seconds8 based on the $30 \mathrm{~km}$ distance and $6 \mathrm{~m} / \mathrm{s}$ wind. This estimated source end time may be adjusted dynamically as the program runs.

8 This choice is arbitrary, however, if the sampler averaging time is the same for all samplers, a smoother numerics results from setting the estimated end time to be an integer multiple of the sampler averaging time 
In Figures 11a through $11 \mathrm{c}$ we observe the continual increase of both the estimated source rate and the predicted downwind concentrations. However, the first peak of the source function is observed at 20 minutes (Fig. 11d), followed by the gradual build-up for the next hour. The large increase step observed in Fig. $11 \mathrm{f}$ is due to the numerics at that particular time. The unit response matrix has only a small portion of the sampler integration time thus requiring a high source rate to match the observation. Note that in Figs. $11 \mathrm{~g}$ and $11 \mathrm{~h}$ this sudden large step is no longer observed. Fig. 14 which compares the total mass released as a function of time for differing estimates of source end time also shows that the overshoot of Fig. $11 \mathrm{f}$ is due to numerics.
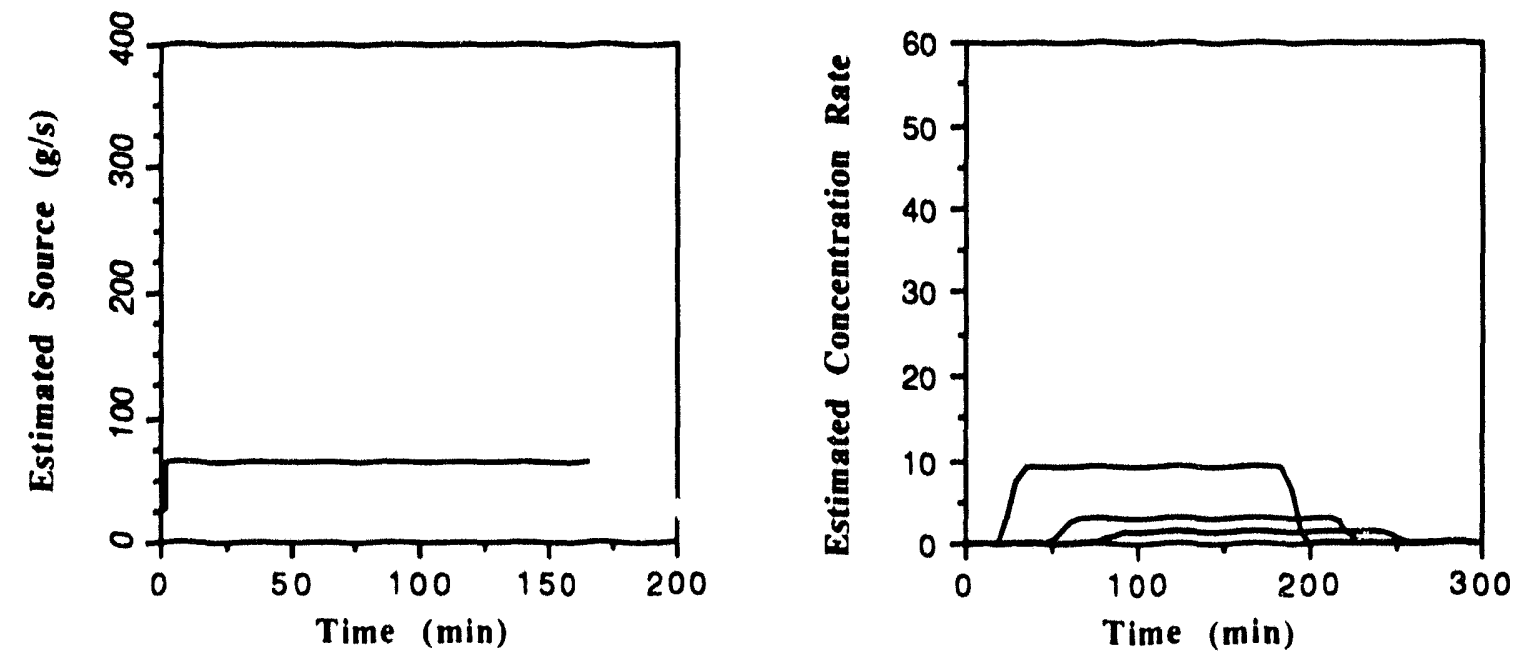

Figure 11a: Estimations at 5 minutes using 2 data points.
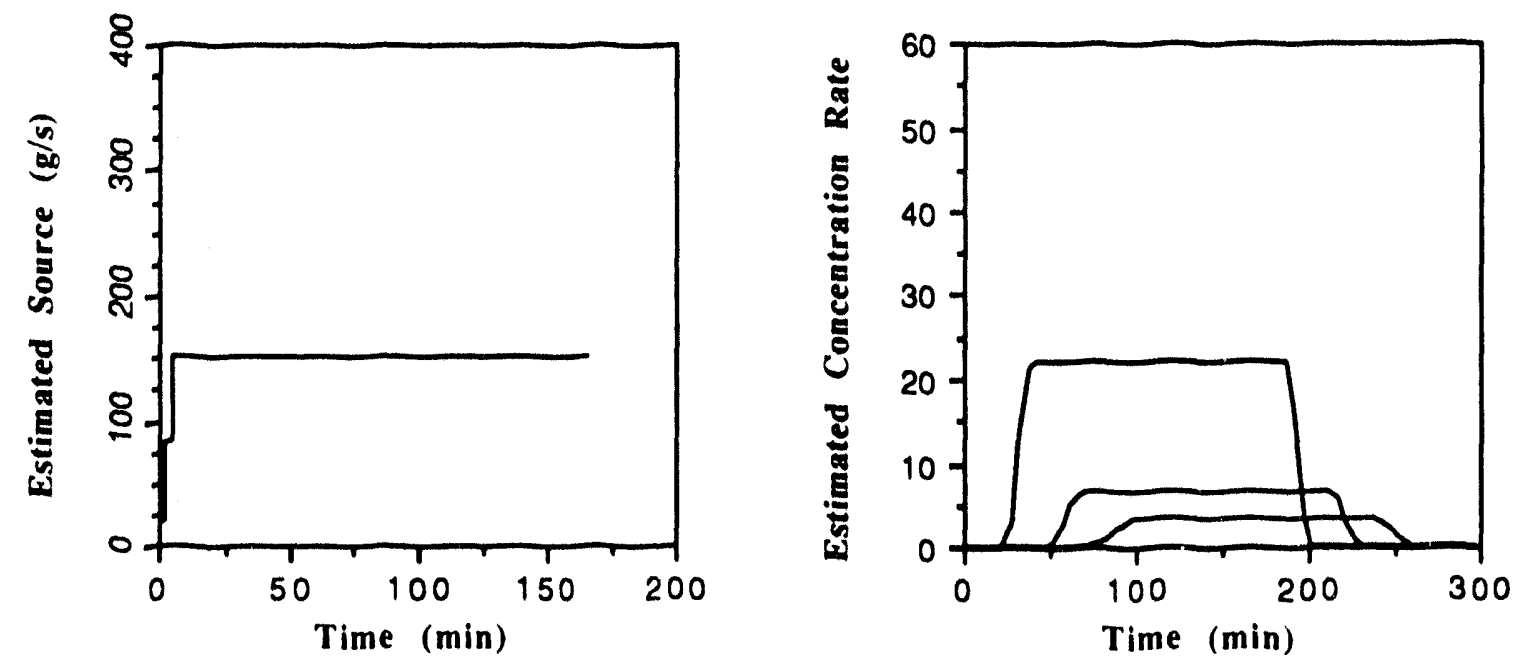

Figure 11b: Estimations at 10 minutes using 4 data points. 
Fig. 11i at 120 minutes since the beginning of the accident the source estimation has captured the second peak of the release. At 150 minutes, Fig. 11 , one observes that the release has essentially stopped.
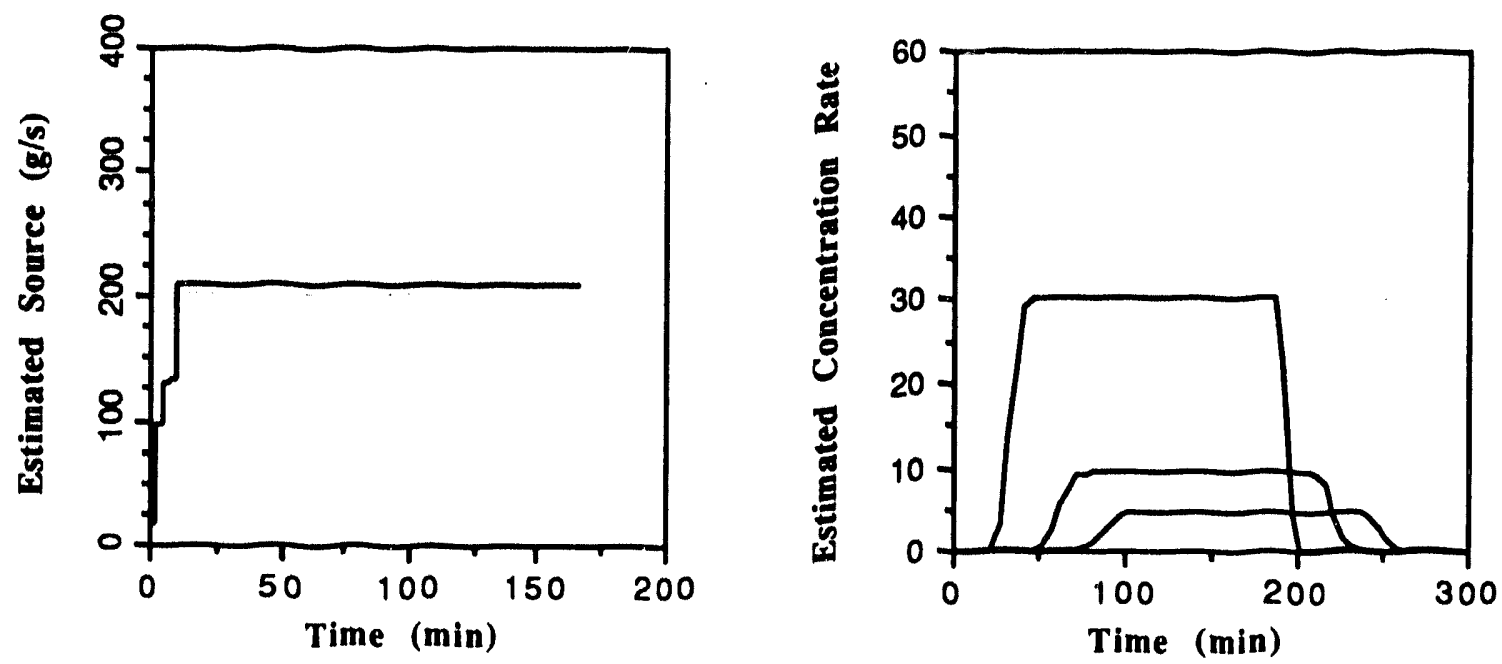

Figure 11c: Estimations at 15 minutes using 6 data points.
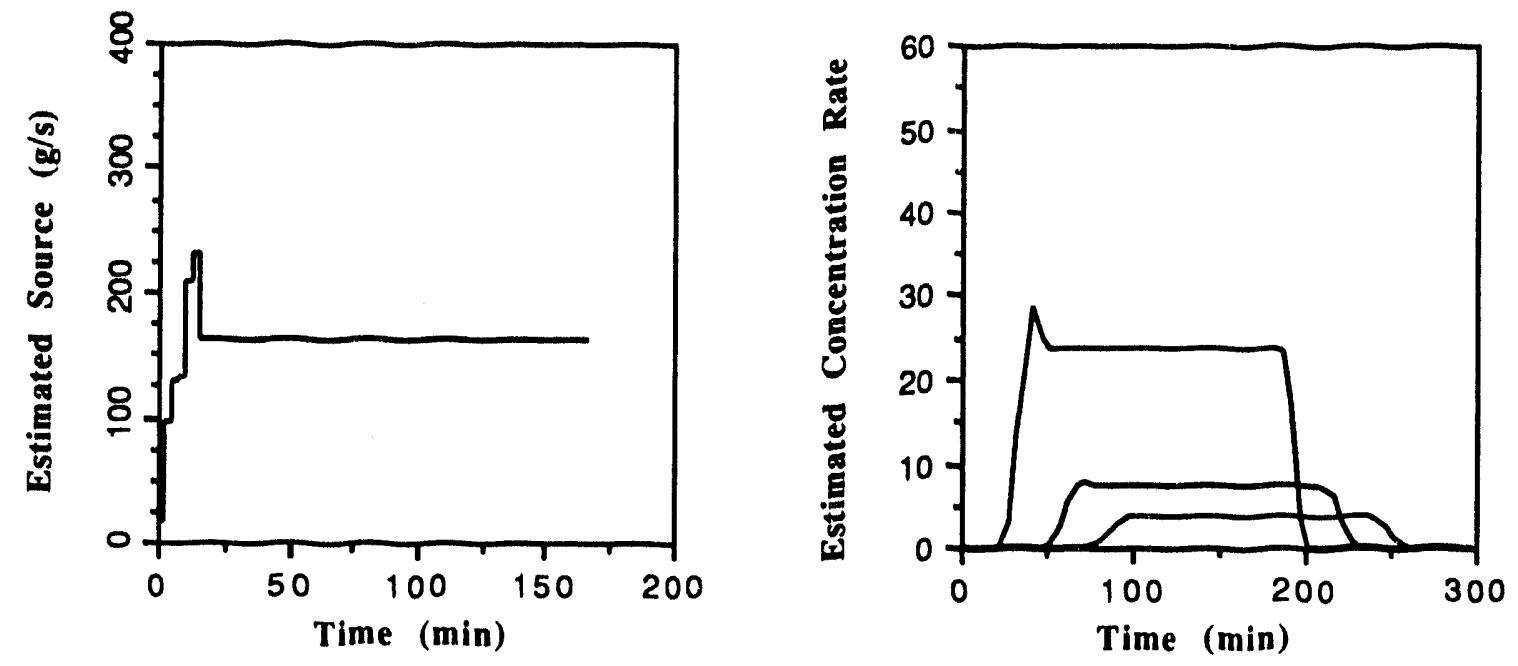

Figure 11d: Estimations at 20 minutes using 8 data points. 
Figs. 12 and 13 are at 155 minutes after the release has been contained. Fig. 12 compares the estimated release rate with the actual (used to generate the data) release function as well as the integrals of the estimated and actual release. The agreement is remarkably good in as much as the data is acquired using 5 minute averages for sample rates and then up to $30 \%$ perturbations are applied. The estimated total mass released in this case is $1.26 \times 10^{6}$ grams, about $10 \%$ higher than the $1.15 \times 10^{6}$ grams actually released by the simulated accident.
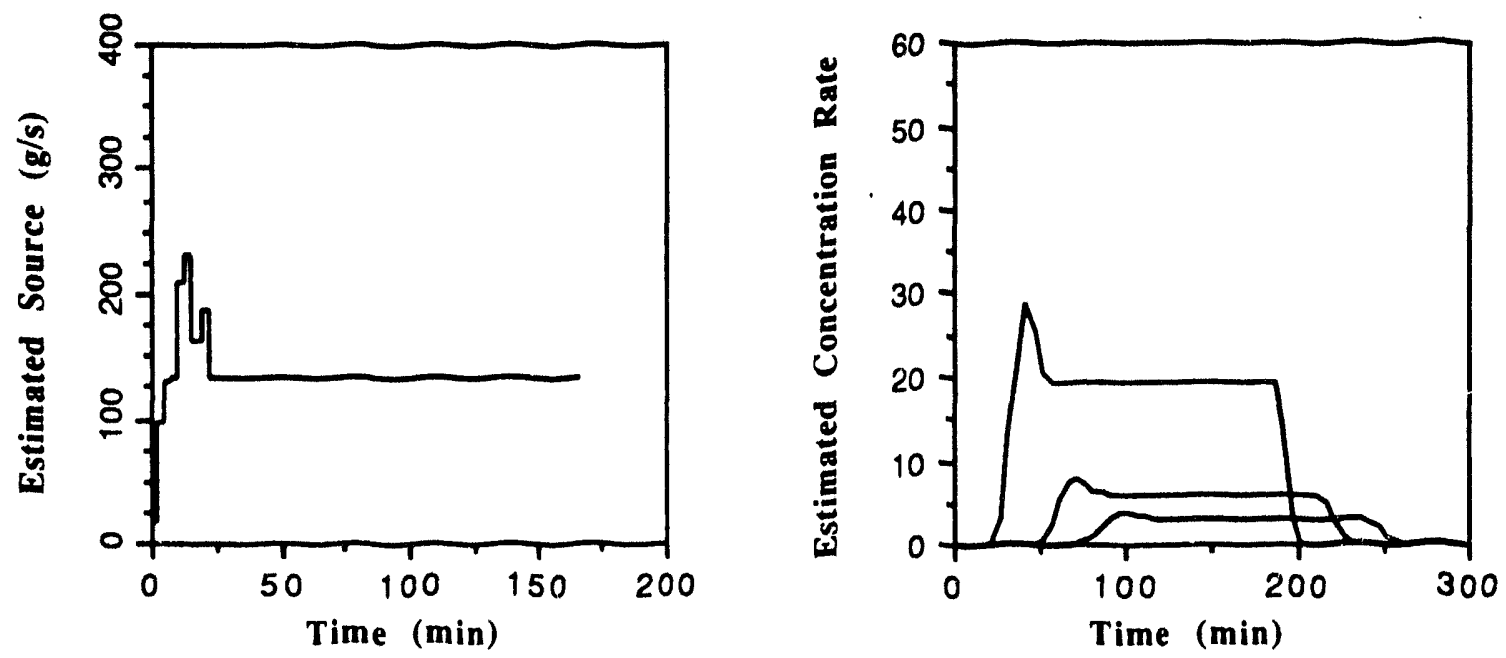

Figure 11e: Estimations at 25 minutes using 10 data points.
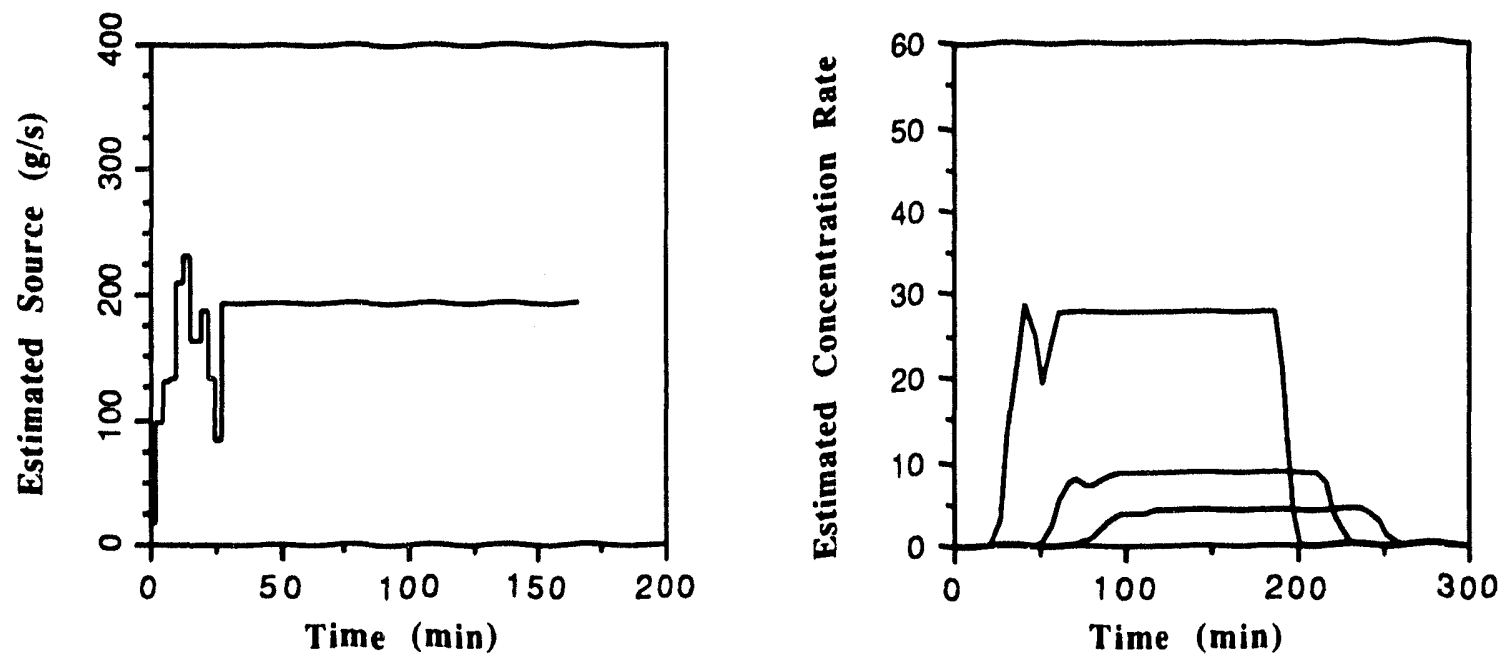

Figure 11f: Estimations at 30 minutes using 12 data points. 
Figure 13 conipares the 5 minute averaged concentration rates predicted by the estimated source term with the actual instantaneous rates that the SGP model produces with the source term shown in Fig. 9. Again the agreement is excellent.
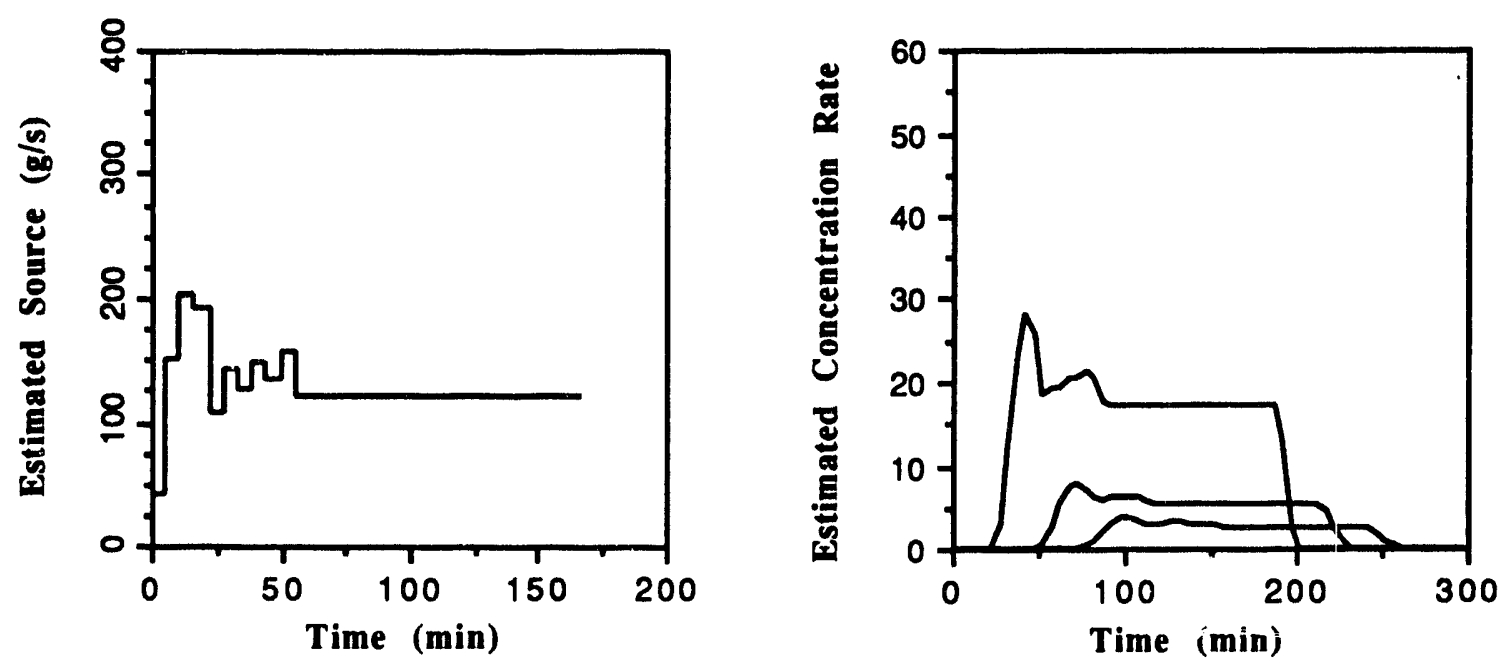

Figure 11g: Estimations at 60 minutes using 24 data pnints.
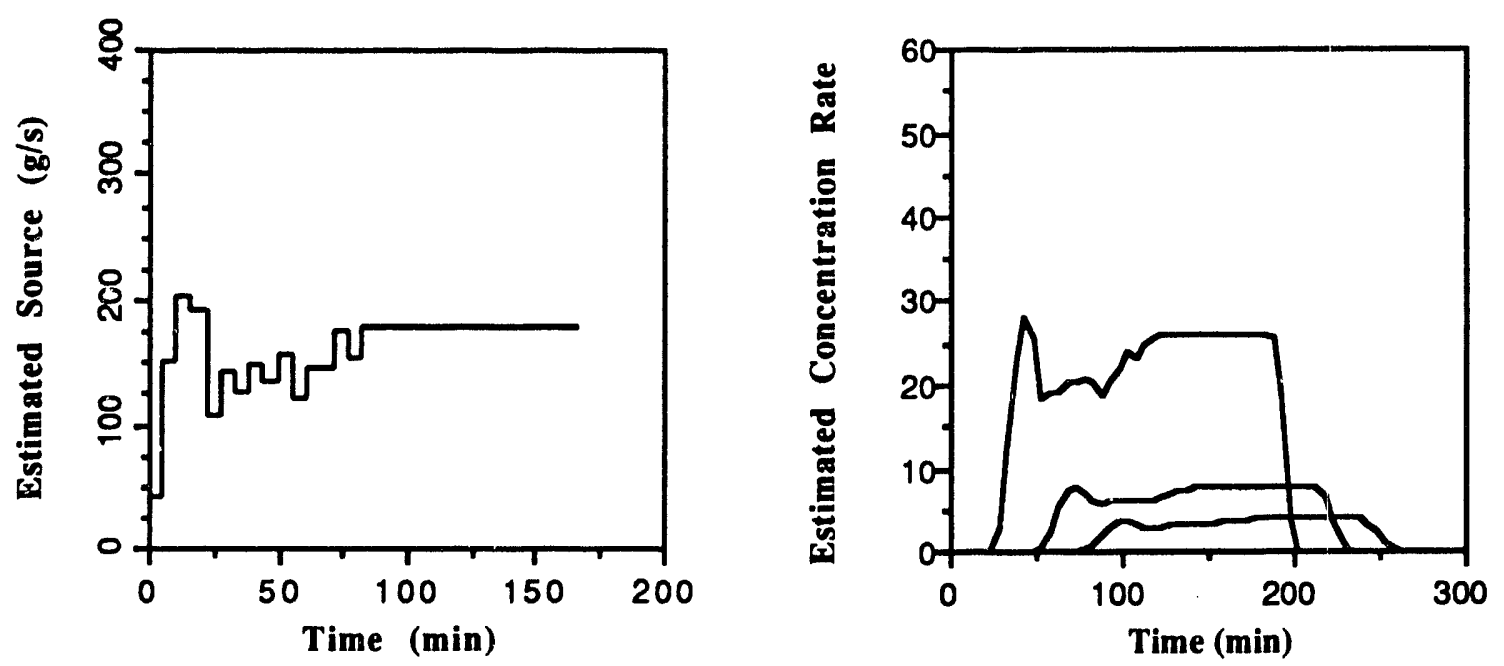

Figure 11h: Estimations at 90 minutes using 36 data points. 
The minimum variation solution uses 60 intervals for the first 30 minutes of estimation then finds the best fit with 30 intervals for the remainder of time. From 35 minutes, Fig. $11 \mathrm{~g}$, through 155 minutes, Fig. 12, the source estimation methodology reproduces the earlier time interval estimates exactly. That is, each additional pair of data points only adjust the last few interval estimates.
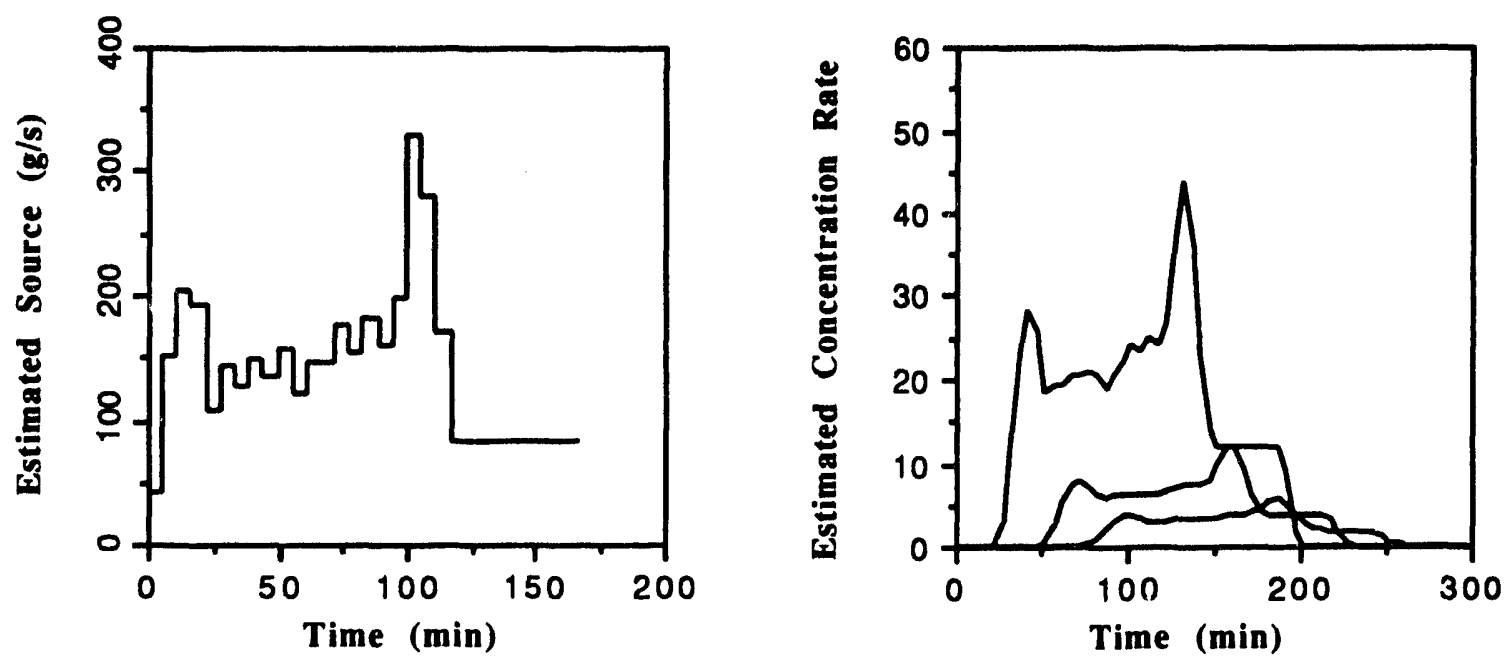

Figure 11i: Estimations at 120 minutes using 48 data points.
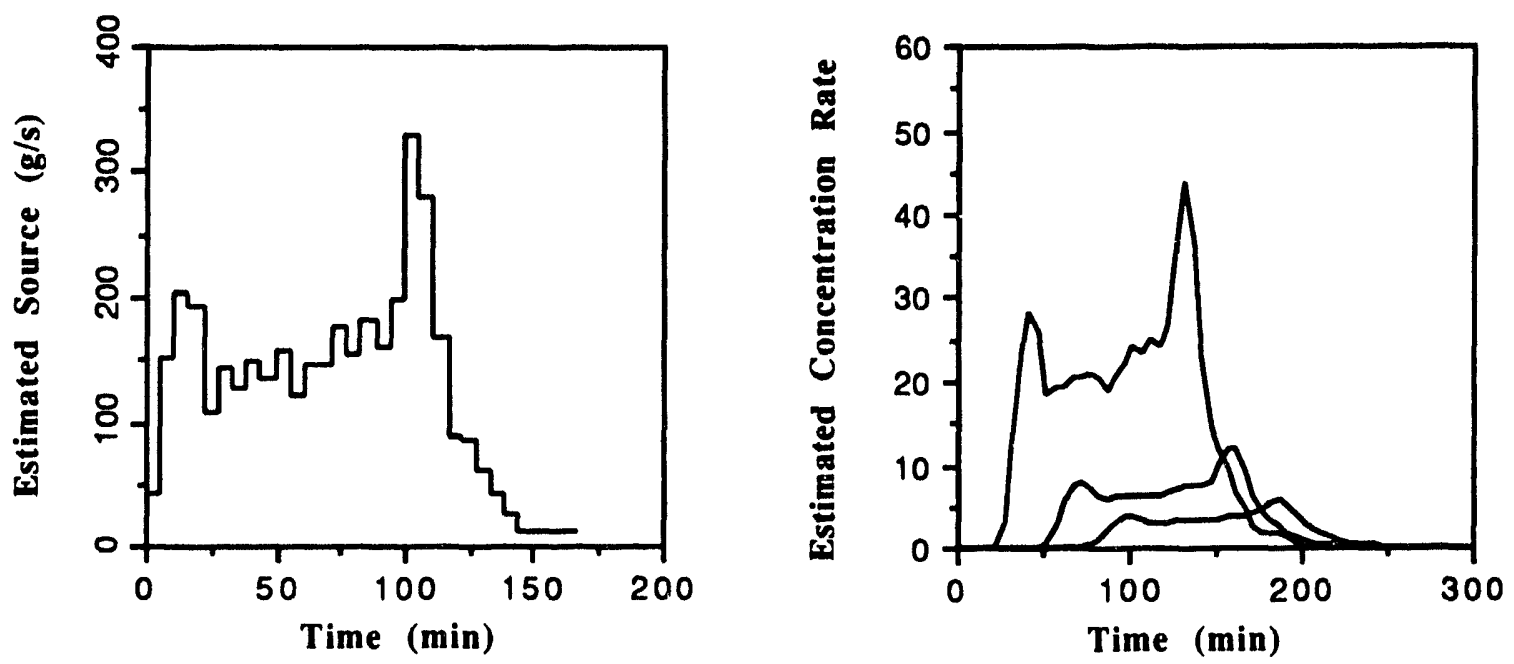

Figure 11j: Estimations at 150 minutes using 60 data points. 

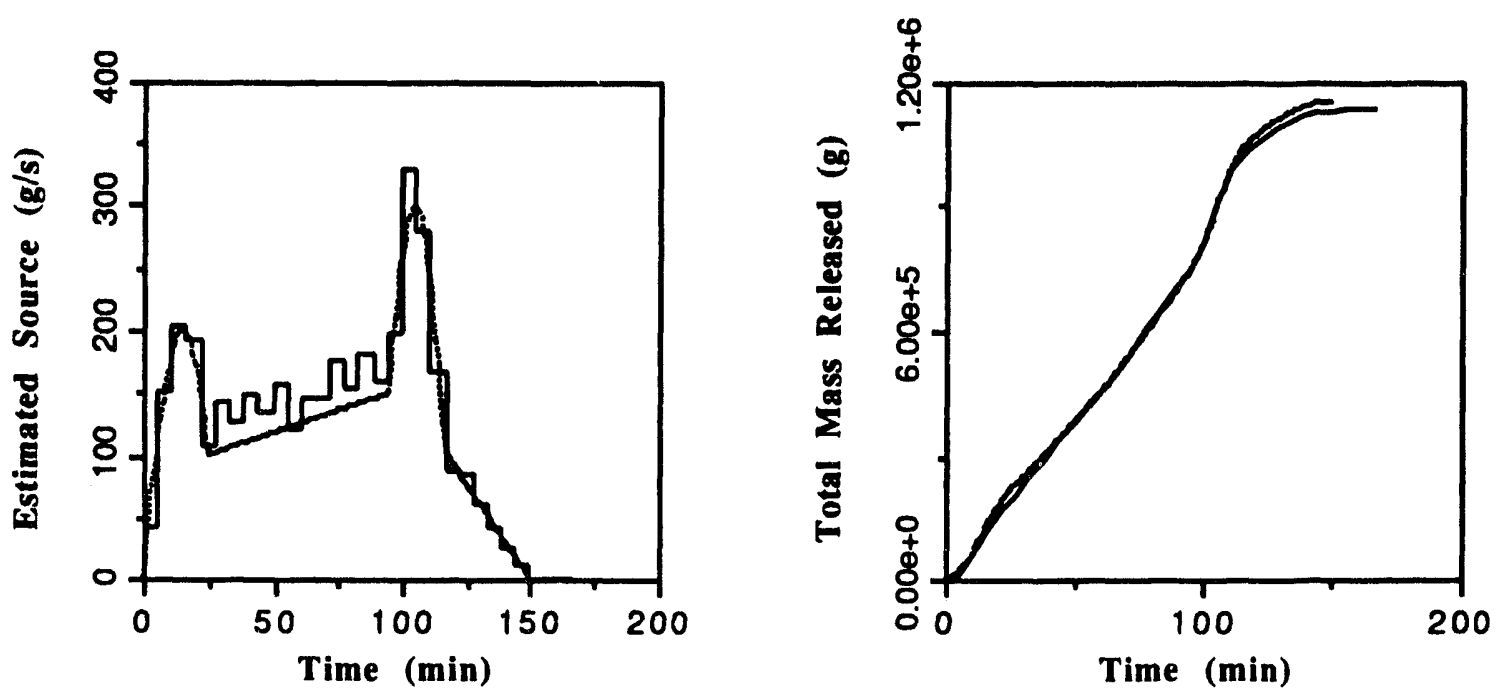

Figure 12: Estimations at 155 minutes using 62 data points. The dotted lines are the actual release function and its integral 


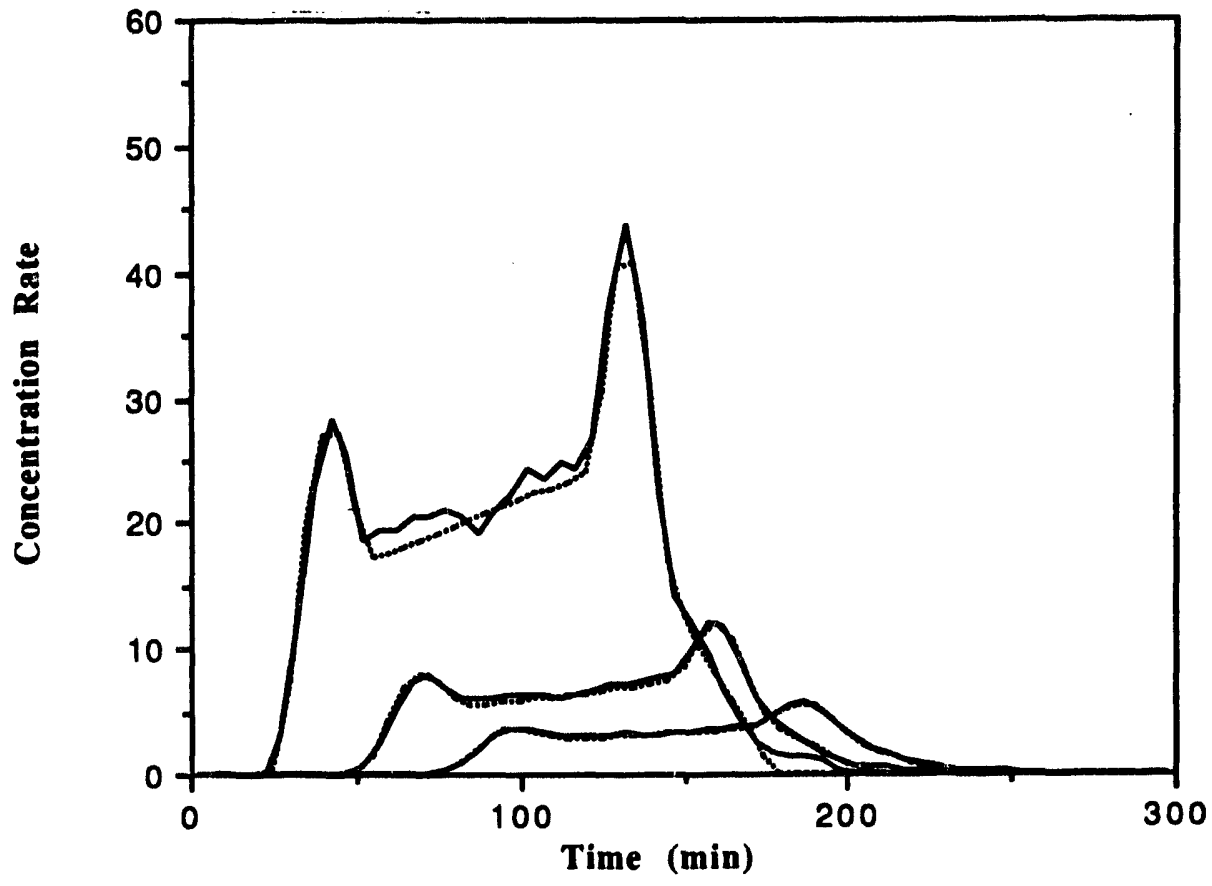

Figure 13: The predicted concentration rates at 10,20 and $30 \mathrm{~km}$ downwind at 155 minutes. The dotted lines are the actual rates.

At this point in time, 155 minutes after the start of the accident, we have provided an estimation of the release function and the possible downwind consequences that is extremely good. Clearly the estimation improves with each additional pair of data points.

The source estimation procedure is extremely fast ${ }^{\mathrm{h}}$ and basically only limited by the computer run time of the atmospheric transport and dispersion model. The only other limitation in terms of real-time emergency response is that of data acquisition and transmission. For our purposes here we have assumed that the data are available to the estimation program at the instant they are measured.

h Using our SGP model on a VAX 6610 in a time-sharing mode, each real time (5 minute interval) source rate estimation required about 30 seconds of wall-clock-time. 


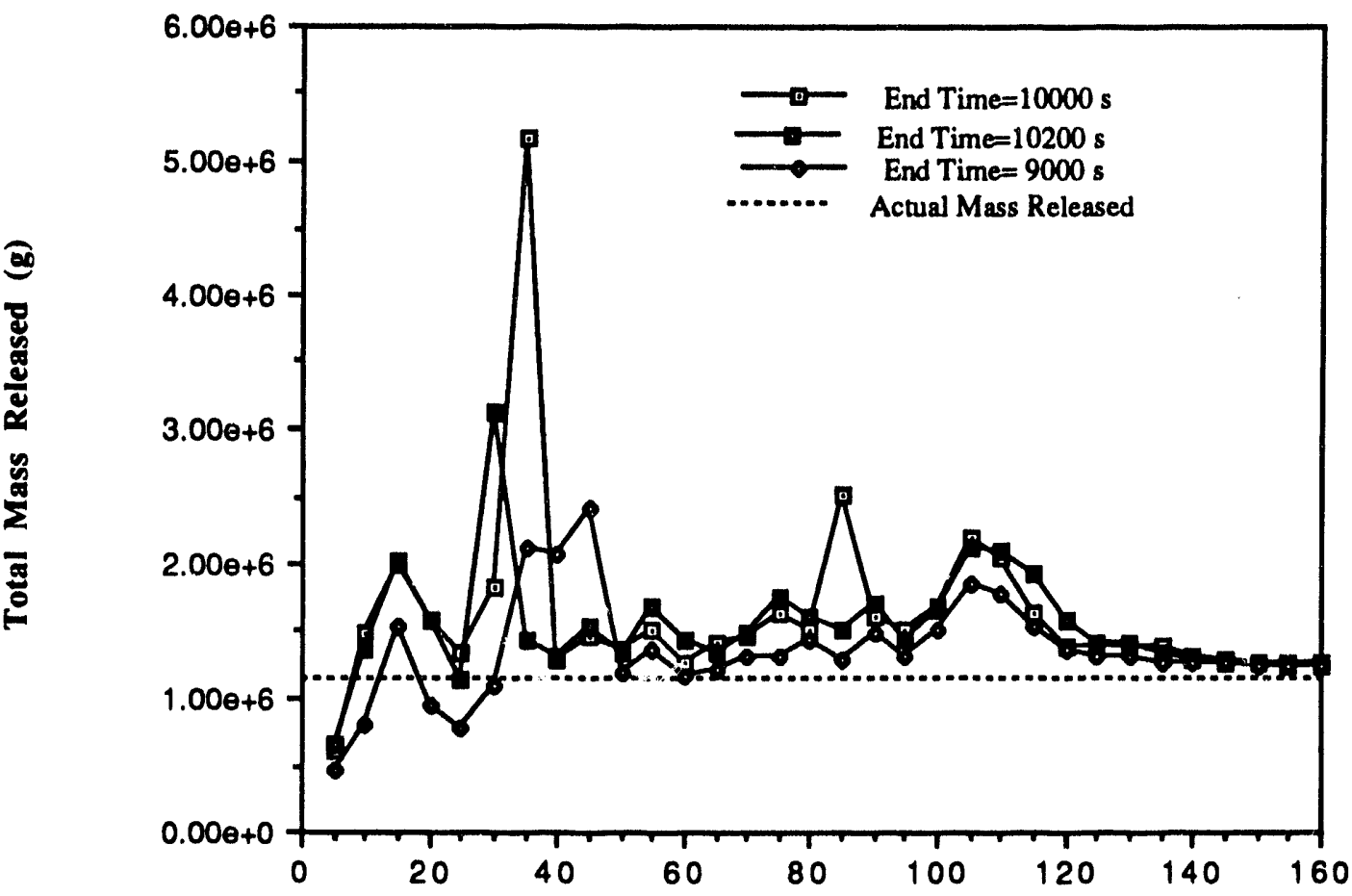

Time (Min)

Figure 14: The estimated total mass released as a function of time compared with the actual release. The differing curves are for "guessed" input values for the release durations of 10000 , 10200 , and $9000 \mathrm{~s}$.

Let us now discuss the uncertainties (or confidence) in the source rate estimation procedure. First, we observed in Fig. 11f, a seemingly large increase in a single interval of the source estimation. Is this real or a numerical artifact? How do we know if we are doing a real time emergency response calculation? Note that with the addition of more data the sudden increase is reevaluated to be much less. Although we have not shown the 35 minute estimation, already by this time the correction has been made. Figure 14 shows the estimated total mass release as a function of time based on the data available at that time. The large increase is evident at 35 minutes with an additional apparent anomaly at 85 seconds. Clearly we would consider these spikes as anomalies since the curves essentially smooth out in time. To further demonstrate the numerical anomaly, we ran two estimations with the estimated end time set at multiples of the sampler averaging period. Observe in Figure 14 the more smooth results on total mass released.

Second, one can consider the uncertainty of the parameter estimations. If the model is "perfect" and the errors in the data are Gaussian, then (c.f., 18,19,20,21,22,23) one can construct the so-called variance/covariance matrix and infer uncertainties and confidence levels. However, since our data errors are only a small part of the uncertainty associated with atmospheric transport and diffusion modeling, we prefer to leave our confidence levels to a heuristic understanding. In particular, for our example, as more data becomes available, not only do our step-function estimation of release repeat the parameter choices, but the downwind predictions 
tend to repeat. We believe that the repetitive nature of the predictions adds to the level of confidence.

Third, the most uncertain aspects of the emergency response model have to do with the atmospheric conditions estimated by the assessor, and how well the transport and diffusion model does when compared with the reality of the conditions during the accident. Table 3 makes some comparisons for the possibility that the diffusion category may be either one too high or one too low. We also compare some cases with wind speeds that are chosen to be different than the actual $6 \mathrm{~m} / \mathrm{s}$ used to simulate the accident. These cases use 64 data points, that is, all the data for the first 160 minutes.

Table 3: Estimated total mass released and predicted peak concentrations due to changes in estimated end time for the source, stability category, and average wind speed. Source fit uses the measurements for the first 105 minutes from 0.5 and $1.0 \mathrm{~km}$ downwind.

\begin{tabular}{|c|c|c|c|c|c|c|c|}
\hline Case & $\begin{array}{c}\text { Estimated } \\
\text { Source End } \\
\text { Time (min) }\end{array}$ & $\begin{array}{c}\text { Pasquill- } \\
\text { Gifford }\end{array}$ & $\begin{array}{c}\text { Average } \\
\text { Wind Speed }\end{array}$ & $\begin{array}{c}\text { Estimated } \\
\text { Release } \\
(\mathrm{m} / \mathrm{s})\end{array}$ & \multicolumn{2}{|c|}{$\begin{array}{c}\text { Predicted Peak Concentrations } \\
\left(\mu \mathrm{g} / \mathrm{m}^{2} / \mathrm{s}\right) \text { at downwind distances }\end{array}$} \\
\cline { 6 - 8 } & 150 & $\mathrm{C}$ & 6 & $1.2529 \mathrm{e} 6$ & 43.42 & 11.95 & 5.588 \\
\hline 1 & 150 & $\mathrm{~B}$ & 6 & $3.1751 \mathrm{e} 6$ & 10.29 & 2.30 & 0.954 \\
\hline 2 & 150 & $\mathrm{D}$ & 6 & $5.0323 \mathrm{e} 5$ & 73.21 & 24.54 & 12.79 \\
\hline 3 & 150 & $\mathrm{C}$ & 5 & $1.0466 \mathrm{e} 6$ & 40.69 & 11.27 & 5.281 \\
\hline 4 & 150 & $\mathrm{C}$ & 7 & $1.4639 \mathrm{e} 6$ & 41.59 & 12.22 & 5.648 \\
\hline 5 & 150 & $\mathrm{~B}$ & 4 & $2.1126 \mathrm{e} 6$ & 11.65 & 2.38 & 0.923 \\
\hline 6 & 150 & $\mathrm{D}$ & 8 & $6.7048 \mathrm{e} 5$ & 73.06 & 25.21 & 13.33 \\
\hline 7 & 150 & & & & &
\end{tabular}

Consider Cases 1, 2 and 3 where the wind speed is the same but the stability categories differ by 1 . Case 1 is our "best-estimate" to the actual release. The estimated mass and the predicted peak concentrations are within $9 \%$ of the actual (instantaneous) values. The moderately unstable (B) conditions are considerably more diffusive than the slightly unstable (C) conditions, which, in turn, are more diffusive than the neutral (D) conditions.

The effects of the diffusion result in factors of 4 to 6 lower in predicted concentrations for $B$ as compared with $C$ and the factors increase as downwind distance increases. Using the measurements from 0.5 and $1.0 \mathrm{~km}$ the estimated total mass released is increased only by a factor of 3 . Due to the lesser diffusion of $\mathrm{D}$ compared with $\mathrm{C}$, the downwind predictions are high by factors of 1.7 to 2.3 and the comparable less estimated mass is lower by a factor of 2.5 .

One can see that if the atmospheric stability conditions are off by as much as a single category the predictions and estimations may be off by about a factor of 5 .

Cases 1, 4 and 5 are all with $C$ stability but the average wind speed increased and decreased by $1 \mathrm{~m} / \mathrm{s}$. In this case we observe only small changes in the predicted concentrations and estimated mass released. That is, reasonable guess of wind speed would not appear to be a serious problem although the estimated mass may be off by about $25 \%$.

The remaining cases 6 and 7 are for B and D stabilities with wind speeds more in keeping with those conditions. The above comments are consistent for these cases also. For example, compare case 2 with case 6 and case 3 with case 7. 
For emergency response purposes, with no a priori knowledge of the actual source rate, we have at our disposal several measures of "goodness of fit" to the data to aid in establishing our confidence level. We have already mentioned the variance/covariance matrix which is applicable only to data errors. In terms of the atmospheric transport and diffusion errors, we may consider the least-squares measure of Eq. 14. For our cases $1,2, \ldots, 7$, the minimum $R_{\varphi}$ 's are 9.2, 54.3, 43.1, 12.1, 9.3, 58.5, 41.9. With this information one can determine that Case 1 gives the best fit to the data.

A second commonly used measure for data fitting is the F factor (often called the factor of $R$ ) where for the ith data point

$$
F_{i} \equiv \begin{cases}o_{i} / c_{i} & \text { provided } c_{i} \geq o_{i}, \text { or } \\ c_{i} / o_{i} & \text { otherwise. }\end{cases}
$$

One then compares the percent of measurements within the F factor, the higher the percentage, the better the fit.
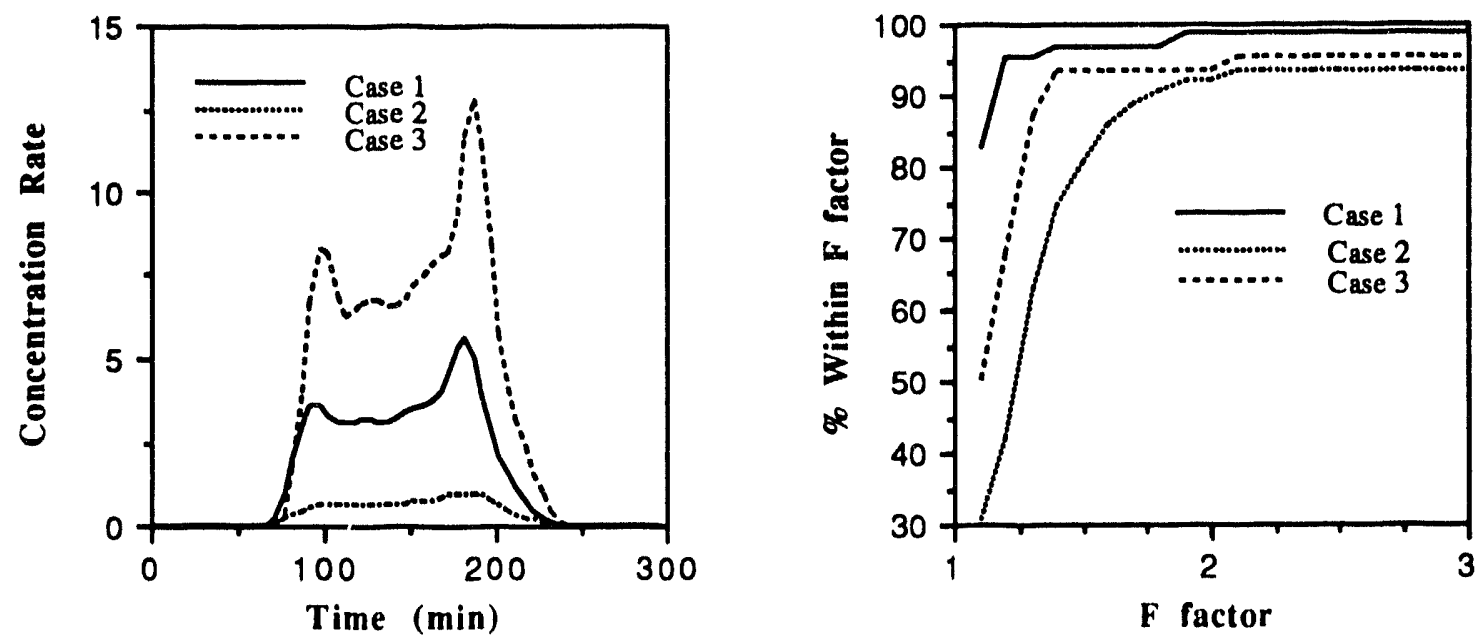

Figure 15: Downwind predicted concentrations and data $F$ factors for Cases 1, 2 and 3 with stability categories C, B and D all with $6 \mathrm{~m} / \mathrm{s}$ wind speed. 

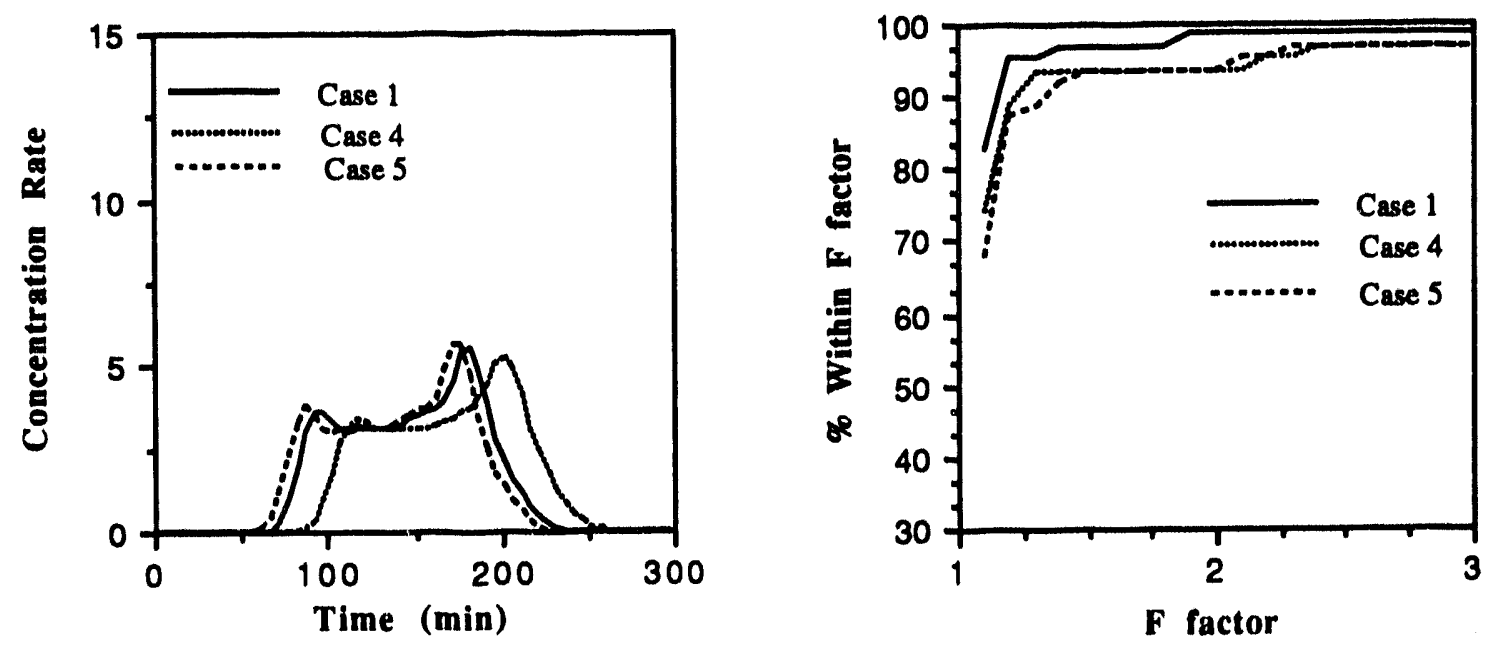

Figure 16: Downwind predicted concentrations and data $F$ factors for Cases 1,4 and 5 with wind speeds of 6,5 and $7 \mathrm{~m} / \mathrm{s}$
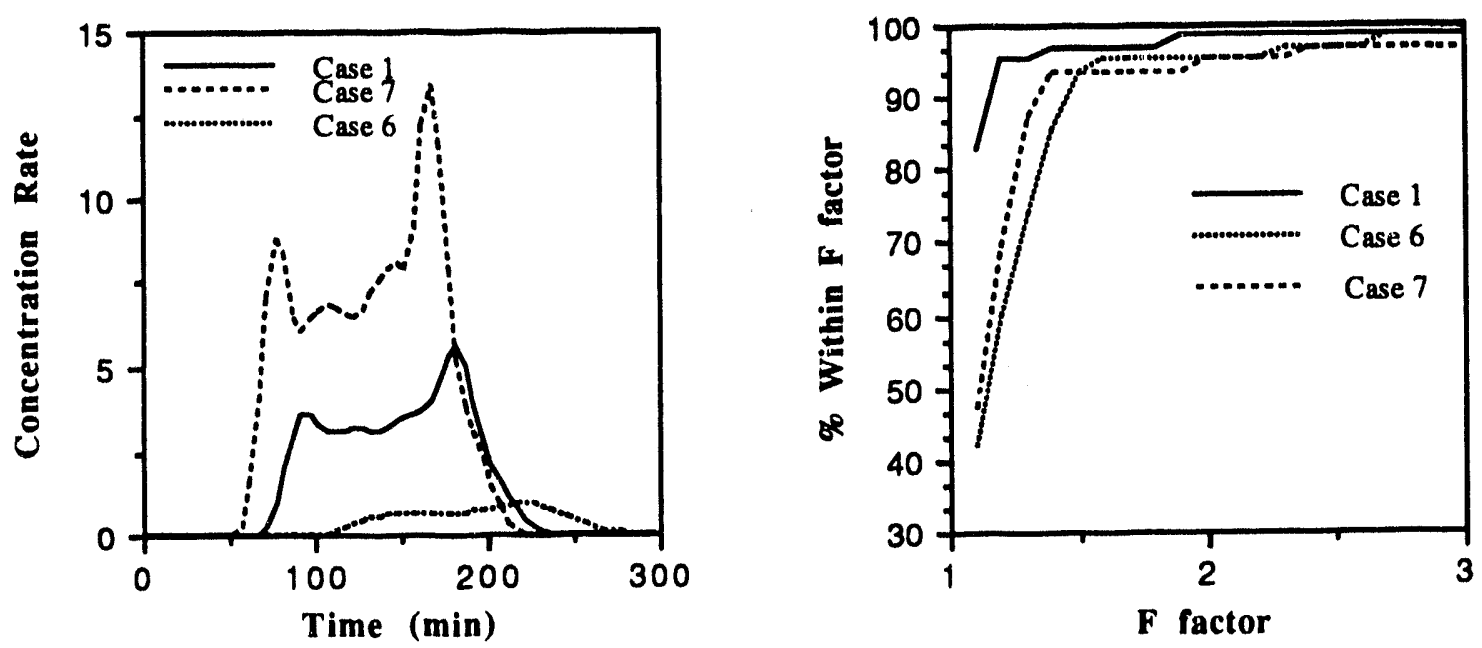

Figure 17: Downwind predicted concentrations and data $\mathrm{F}$ factors for Case 1 with $\mathrm{C}$ stability and $6 \mathrm{~m} / \mathrm{s}$ wind speed, Case 6 with B stability and $4 \mathrm{~m} / \mathrm{s}$ wind speed, and Case 7 with D stability and $8 \mathrm{~m} / \mathrm{s}$ wind speed. 
Figures 15, 16 and 17 compare the predicted downwind concentrations and the $F$ factor plots for the 7 cases. In Fig. 15 it is obvious that Case 1 estimation is best since the $F$ factor curve is clearly above the other two cases. It is also clear that the D stability (Case 3 ) provides a better fit than does the B stability (Case 2). We have already noted that Case 3 is only off by about a factor of 2 whereas Case 2 is off by about a factor of 5 .

The $1 \mathrm{~m} / \mathrm{s}$ error in wind speed is not very dramatic as shown in Fig. 16 and all the fits are reasonable. Fig. 17 reflects similar results as shown by Fig 15.

We have not discussed the effects of errors in wind direction on the results. This topic has been covered in (6) where we discussed using rotations of the model produced data to attain the highest correlation between non-zero measurements and non-zero computed values. That is, we find the largest number of consistent computed measurements as discussed in Section 3.

\section{Conclusions}

With reference to Figures 6,7 and 8 , although we have not shown the data here, if the $30 \%$ accuracy of the samplers is reduced to zero, then the 1 second averages follow the actual release functions extremely well for distances where the structure is not destroyed by atmospheric diffusion. Over all, the numerical algorithms do excellent estimations. The accuracy depends primarily on the accuracy of the instrumentation for data acquisition. That is, for emergency response situations, the accuracy of our predictions will heavily depend on the accuracy and extent of the data available to use for the prediction.

We have demonstrated how the methodology might be ured for aids to decision making in an emergency response situation following an accidental release of toxic material in the atmosphere. We stress that the source fitting function is extremely fast and only limited by the rate of data acquisition, its availability to the model, and the time required for the atmospheric transport and diffusion model to compute the unit response matrix.

We have heuristically discussed the uncertainty and confidence of the model estimations and shown how one gains confidence in the results. Certainly it would be possible to attempt to propagate probability distributions through the models to gain confidence bounds. However, such "probability analysis" requires setting initial probability functions of each of the model parameters such as source rate, data measurements, atmospheric conditions, wind-speed, etc., clearly an area that is not well-known with any great confidence. 


\section{References}

1. Proceedings of the ANS Topical Meeting on Emergency Response--Planning, Technologies, \& Implementation, COI $\sqrt{F-880913, ~ U C-610, ~ C h a r l e s t o n, ~ S C, ~ S e p t e m b e r ~ 26-~}$ $28,1988$.

2. Proceedings Workshop on Real-Time Computing of the Environmental Consequences of an Accidental Release To Atmosphere From a Nuclear Installation, Doc. Nr. V 2943/86 En, Fr, Commission of the European Communities, Luxembourg, September 17-20, 1985

3. Proceedings of Second International Workshop on Real-Time Computing of the Environmental Consequences of An Accidental Release to the Atmosphere from a Nuclear Installation, "Decision Aids to Off-Site Emergency Management," Commission of the European Communities, Luxembourg, May 16-19, 1989

4. Slade, D.H., 1968, Meteorology and Atomic Energy 1968, U.S. Atomic Energy Commission of Information Services, $445 \mathrm{pp}$.

5. Gudiksen, P.H., T.F. Harvey, and R. Lange, 1989, "Chernobyl Source Term, Atmospheric Dispersion, and Dose Estimation," UCRL-98235 Rev. 2, Preprint, Lawrence Livermore National Laboratory, Livermore, CA USA 94550.

6. Edwards, L.L., R.P. Freis, L.G. Peters, P.H. Gudiksen, and S.E. Pitovranov, 1993, "The Use of Nonlinear Regression Analysis for Integrating Pollutant Concentration Measurements with Atmospheric Dispersion Modeling for Source Term Estimation," Nucl. Technol., 101 (2), 168 (Also UCRL-JC-108978-Rev. 1, Lawrence Livermore National Laboratory, Livermore, CA USA ).

7. Zannetti, P., 1990, "Air Pollution Modeling: Theories, Computational Methods and Available Software," Van Nostrand Reinhold, New York, USA.

8. Rodriguez, D.J., G.D. Greenly, P.M. Gresho, R. Lange, B.S. Lawver, L.A. Lawson, and H. Walker, 1982,"User's Guide to the MATHEW/ADPIC Models," UCRL-MA-103581, Lawrence Livermore National Laboratory, Livermore, CA USA 94550.

9. Sherman, C.S., 1978, "A Mass-Consistent Model for Wind Fields Over Complex Terrain," J. Appl. Meteor., 17, 312-319.

10. Lange, R., 1978, "ADPIC-A Three-Dimensional Particle-in Cell Model for the Dispersal of Atmospheric Pollutants and Its Comparison to Regional Tracer Studies," J. Appl. Meteor., 17, 320-329.

11. Dickerson, M.H., 1984, "Chapter 7: Atmospheric Dispersion and Dose Calculation Techniques," UCRL-90765 preprint, Lawrence Livermore National Laboratory, Livermore, CA USA 94550.

12. Groetsch, C.W., 1984, "The theory of Tikhonov regularization for Fredholm Equations of the first kind." Boston, Pitman Publ. Ltd.

13. Kress, R., 1989, "Linear Integral Equations," Applied Mathematical Sciences, 82, SpringerVerlag, New York, 299pp. 
14. Hadamard, J., 1902,"Sur les problemes aux derivees partielles et leur physique," Bull. Princeton Univ., 13.

15. Tikhonov, A.N., and V.Y. Arsenin, 1977, "Solution of Ill-Posed Problems," Translation Editor F. John, V.H. Winston \& Sons, Washington, D.C., 258 pp.

16. Andersen, R.S., F.R. de Hoog, and M.A. Lukas, 1980, "The application and numerical solution of integral equations." Sijthoff \& Noordoff, Germantown, Maryland, USA, 259 pp.

17. Baker, C.T.H., and G.F. Miller, Eds., 1982, "Treatment of Integral Equations by Numerical Methods," Basc J on the Proceedings of a Symposium held in Durham for 19-29 July, 1982, organized under the auspices of the London Mathematical Society, Academic Press, New York, $493 \mathrm{pp}$.

18. Dabbert, W.F., and R.N. Dietz. 1986 "Gaseous Tracer Technology and Applications" in Probing the Atmospheric Boundary Layer, D.H. Lenschow, Ed., Amer. Met. Society, Boston, MA.

18. Bevington, P.R., 1969, Data Reduction and Error Analysis for the Physical Sciences, McGraw-Hill, NY, $336 \mathrm{pp}$.

19. Bard, Y., 1974, Nonlinear Parameter Estimation, Academic Press, NY. 341 pp.

20. Tarantola, A., 1987, "Inverse problem theory," Elsevier Science Pub. Neth. 601 pp.

21. Marquardt, D.W., 1963, "An Algorithm for Least-Squares Estimation of Nonlinear Parameters," J. Soc. Ind. App. Math. 11, 431-441

22. Atkinson, K.E., 1989, An Introduction to Numerical Analysis, 2nd Ed. John Wiley \& Sons, NY, $693 \mathrm{pp}$.

23. Lawson, C.L., and R.J. Hanson, 1974, Solving Least Squares Problems, Prentice-Hall, inc., Englewood Cliffs, NJ. 340pp. 

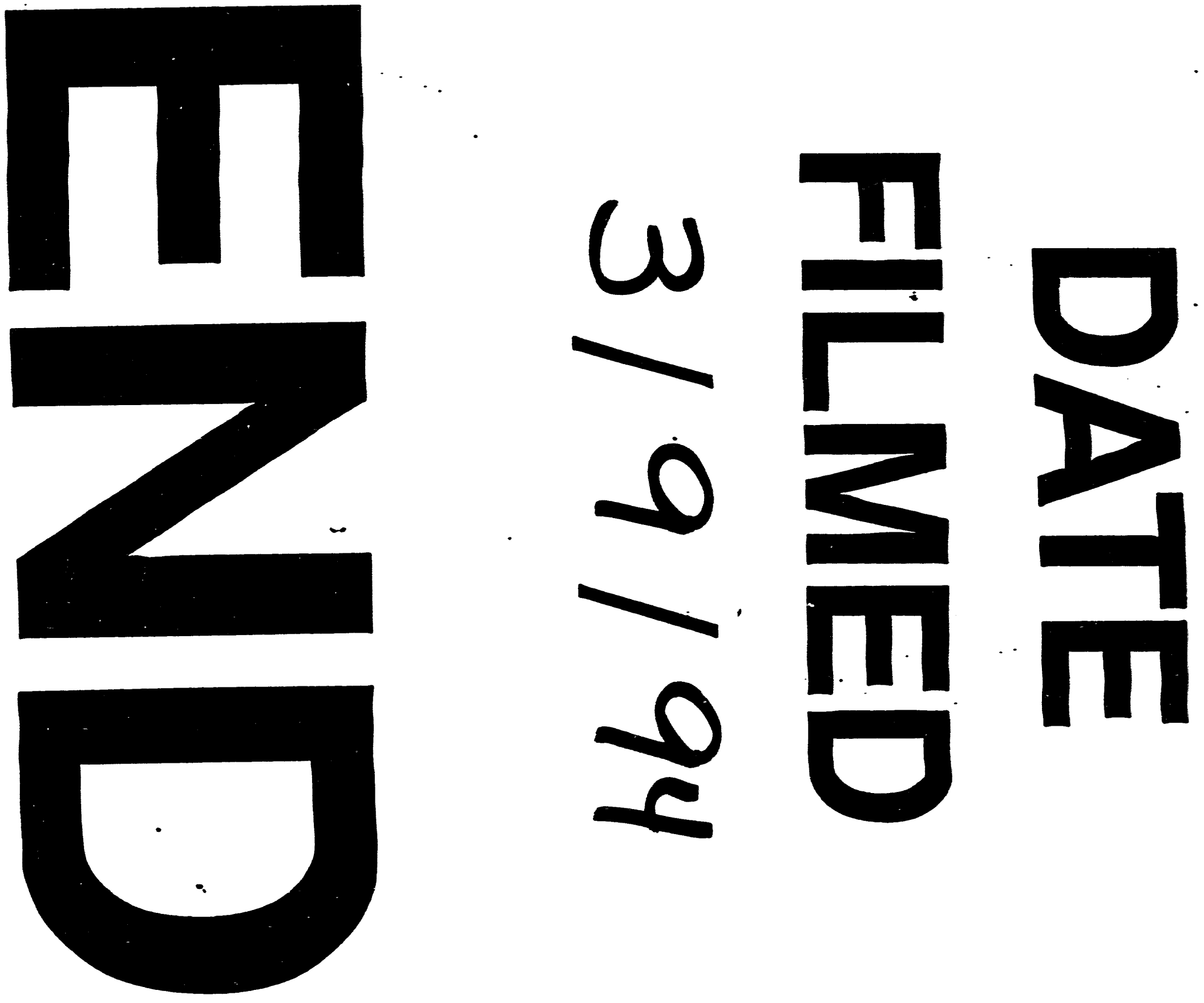
